\title{
A Network Pharmacology-based Approach to Explore the Active Ingredients and Molecular Mechanism of Lei-gong-gen Formula Granule on a Spontaneously Hypertensive Rat Model
}

Qiaofeng Li

Guangxi Medical University

\section{Taijin Lan}

GuangXi University of Chinese Medicine

\section{Songhua He}

Guangxi Institute for Food and Drug Control

\section{Weiwei Chen}

Guangxi Medical University

\section{Xiaolan Li}

Guangxi Medical University

\section{Weiquan Zhang}

Guangxi Medical University

\section{Ying Liu}

Guangxi Medical University

\section{Qiuping Zhang}

Guangxi Medical University

\section{Xin Chen}

Guangxi Medical University

\section{Yaoyao Han}

Guangxi Medical University

\section{Zhiheng Su}

Guangxi Medical University

\section{Dan Zhu}

Guangxi Medical University

Hongwei Guo ( $\square$ hongweiguo@gxmu.edu.cn )

Guangxi Medical University https://orcid.org/0000-0002-4046-2643

\section{Research}


Keywords: Centella asiatica (L.) Urb., Eclipta prostrata (L.) L., Smilax glabra Roxb., hypertension, network pharmacology

Posted Date: March 2nd, 2021

DOI: https://doi.org/10.21203/rs.3.rs-253571/v1

License: (c) (i) This work is licensed under a Creative Commons Attribution 4.0 International License. Read Full License 


\section{Abstract}

\section{Background}

Lei-gong-gen formula granule (LFG) is a folk prescription derived from Zhuang nationality, which is the largest ethnic minority among the 56 nationalities in China. It consists of three herbs, namely Eclipta prostrata (L.) L., Smilax glabra Roxb, and Centella asiatica (L.) Urb. It has been widely used as health protection tea for hundreds of years to prevent hypertension in Guangxi Zhuang Autonomous Region. The purpose of this study is to validate the antihypertensive effect of LFG on a SHR model and further to identify the active ingredients of LFG and its anti-hypertension molecular mechanism.

\section{Methods}

Firstly, a spontaneously hypertensive rat model was used to observe the effects of LFG on blood pressure,

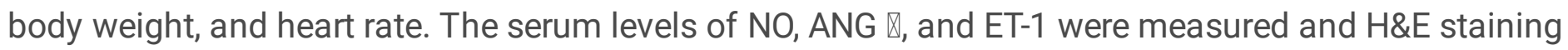
was applied to observe the pathology of the heart. Secondly, network pharmacology analysis was performed to collect the ingredients of LFG by using the database of traditional Chinese medicine (TCMSP, TCMID, BATMAN-TCM) and to predict the active compounds, their corresponding targets, and hypertension associated pathways. Then the predicted results were verified by molecular biology experiments such as RT-qPCR and western blot. Finally, the potential active compounds were predicted by molecular docking technology, and the LFG chemical constituents were analyzed and identified by UPLCQTOF/MS technology.

\section{Results}

LFG significantly reduced blood pressure and increased serum NO content in SHR rats. LFG ameliorated the pathological changes such as cardiac hypertrophy and interstitial inflammation. Based on the results of public database searching, 53 candidate active compounds from LFG were collected, which link to 765 potential targets. 828 hypertension associated targets were retrieved. 12 overlapped targets both related to candidate active compounds from LFG and hypertension were screened and used as the potential targets of LFG on antihypertensive effect. And the 12 overlapped targets were validated using RT-qPCR, and the results showed that LFG could upregulate the mRNA expression of NOS3 and proto-oncogene tyrosine-protein kinase SRC (SRC) in the thoracic aorta. Pathway enrichment results showed that PI3KAKT signaling pathway was closely related to the expression of NOS3 and SRC. To verify this result, western blot was used to detect the key proteins (PI3K, AKT, and p-AKT) of the PI3K-AKT signaling pathway. The results showed that LFG significantly increased the protein expression levels of PI3K and phosphorylated AKT in SHR rats, suggesting that LFG may active PI3K-AKT signaling pathway to decrease hypertension. Molecular docking study further supported that p-hydroxybenzoic acid, cedar acid, shikimic acid, salicylic acid, nicotinic acid, linalool, and histidine can be well binding with NOS3, SRC, 
PI3K, and AKT. UPLC-QTOF/MS analysis confirmed that p-hydroxybenzoic acid, shikimic acid, salicylic acid, and nicotinic acid existed in LFG.

\section{Conclusion}

LFG can reduce the blood pressure of SHR rats, which might be attributed to increasing the serum NO level for promoting vasodilation via upregulating SRC expression level and activating the PI3K-AKT-NOS3 signaling pathway. p-Hydroxybenzoic acid, shikimic acid, salicylic acid, and nicotinic acid might be the underlying compounds for LFG antihypertensive effect.

\section{Introduction}

Hypertension is one of the most common cardiovascular diseases, which can cause damage to the heart, brain, kidney, and other important organs [1]. It is an important risk factor for the death of the global population and poses a serious threat to the safety and quality of patients' life. China has conducted large-scale population surveys of hypertension, and these surveys showed consistently a rapidly increasing trend in the hypertension prevalence, from $5.1 \%$ in 1959 to $27.8 \%$ in 2014 [2]. In Europe, Canada, and the United States, the prevalence is reported to vary from 27-55\% [3]. Hypertension is a chronic disease, and there is no complete cure for high blood pressure. Currently, drug treatment is mainly used to control the level of blood pressure and bring it down to a normal range in clinical practice. Although the existing antihypertensive drugs can effectively control blood pressure in the short term, it is prone to develop adverse effects for the long term taken, which greatly affects their treatment compliance [4]. Therefore, the development of drugs which can effectively lower the blood pressure with fewer adverse reactions is still the main direction of current research. Traditional Chinese Medicine (TCM) has accumulated much experience in the treatment of hypertension [5]. TCM has the characteristics of multicomponent, multi-target, and multi-pathways, especially has a certain effect in improving the complications of hypertension [6]. Thus, screening high-efficiency antihypertensive drugs based on Chinese medicine formula with fewer side effects has attracted considerable attention.

Lei-gong-gen formula granule (LFG) is an effective antihypertensive traditional Chinese folk prescription which is soaked in wine or taken as tea by the local residents in Guangxi Zhuang Autonomous Region for many years [7]. Epidemiological data showed that the prevalence of hypertension in local residents was $13.70 \%$, which was significantly lower than the national average (25.2\%) [8]. It is speculated that the lower incidence of hypertension of the local people may be related to their long-term using of this formula. LFG is composed of three herbs, containing Centella asiatica (L.) Urb. (Ji Xue Cao, also known as Lei-gong-gen in Chinese medicine), Eclipta prostrata (L.) L. (Mo Han Lian), and Smilax glabra Roxb. (Tu Fu Ling). Centella asiatica (L.) Urb., a tropical medicinal plant belonging to the family Apiaceae (Umbelliferae), is widely distributed in many parts of the world especially in Asian countries including India, China, and Nepal. Centella asiatica (L.) Urb. was reported to exhibit cardioprotective, antihypertensive, antioxidant, and anti-inflammatory activities [9]. Recent studies showed that asiatic acid 
reduced blood pressure, improved vascular function by restoring endothelial NO synthase (eNOS), and p47phox expression, and alleviates cardiovascular remodeling by restoring iNOS/eNOS expression in LNAME hypertensive rats [10]. Eclipta prostrata (L.) L. is an annual herb of the Asteraceae family, native to China, Japan, and India [11]. Eclipta prostrata (L.) L. was reported to have significant pharmacological features such as antioxidant, anti-inflammatory, and hypotensive activities [12]. Smilax glabra Roxb., is a rhizome of the Liliaceae plant [13]. Pharmacological studies demonstrated that the flavonoids of Smilax glabra Roxb. exhibited anti-cardiac hypertrophy and anti-hypertensive effects on renovascular hypertension rats through regulating the level of atrial natriuretic peptide [14].

Chinese herbal formulae containing a large number of compounds are too complex to be analyzed by traditional experimental methods based on the paradigm of "One gene, one drug, one disease" [15]. Accumulating evidence shows that network pharmacology can elucidate the potential mechanisms of multi-component and multi-target agents by analyzing various complex and multi-level interaction networks [16]. Network pharmacology has become a powerful tool to reveal the functions and behaviors of complex biological systems systematically, and it has become a novel and effective way to explore the pharmacological mechanism of TCM [17].

In this study, we investigated the active ingredients, corresponding targets, and pharmacological mechanisms of LFG antihypertensive effect by integrating network pharmacology prediction and molecular biology experimental validations. As shown in Fig. 1, the whole study consists of three steps. Step 1: animal experiments. A spontaneously hypertensive rat (SHR) model was firstly used to observe the antihypertensive effect of LFG. Step 2: network pharmacology analysis. The active ingredients, corresponding targets, and hypertension associated pathways were predicted by public databases. Protein-protein interaction (PPI) networks of LFG-related and hypertension-related targets were constructed, and core targets were identified through topological analysis. Step 3: experimental verification. The predicted results were verified by molecular biology methods such as RT-qPCR and western blot based on SHR model. The potential active ingredients were predicted by molecular docking technology, and the LFG chemical constituents were analyzed and identified by Ultra performance liquid chromatography/quadrupole time-of-flight mass spectrometry (UPLC-QTOF/MS) technology. The findings of this study may contribute to the clarification of active ingredients and underlying mechanisms of LFG and provide an accurate and reasonable reference of LFG clinical application.

\section{Materials And Methods}

\subsection{Drugs and reagents}

Lei-gong-gen formula granule (LFG) was provided by Guangxi Institute of Chinese Medicine and Pharmaceutical Science (Guangxi, China, lot No. 20170210). The authentication of three dried herbal drugs (Centella asiatica (L.) Urb., Eclipta prostrata (L.) L., Smilax glabra Roxb.) has been specified, and the preparation of LFG was shown in our previous research $[18,19]$. Captopril was obtained from Teyi Pharmaceutical Group Co., Ltd (Guangdong, China, lot No. 20170501). 
Nitric oxide (NO) assay kit was obtained from Nanjing Jiancheng Bioengineering Institute (Nanjing, China, Cat. A013-2). Rat angiotensin $\otimes$ (ANG $\otimes$, Cat. CSB-E04494r) and rat endothelin 1 (ET-1, Cat. CSB-E06979r) ELISA kits were obtained from CUSABIO (Wuhan, China). Trizol reagent (Cat. 15596026), cDNA Reverse Transcription Kit (Cat. 4368814), SYBR Green Master Mix (Cat. A25742), Pierce Protease and Phosphatase Inhibitor Mini Tablets (Cat. A32961), RIPA buffer (Cat. 89900), Prestained Protein Ladder (Cat. 26616), Tween 20 (Cat. 85113), Chemiluminescent Substrate (Cat. 24580) were purchased from Thermo Fisher Scientific (Waltham, USA). The Bicinchoninic acid (BCA) Protein Assay Kit (No. P0011) was purchased from Beyotime Biotechnology Co., Ltd. (Shanghai, China).

GAPDH (14C10) Rabbit mAb (Cat. 2118), PI3 Kinase p110a (C73F8) Rabbit mAb (Cat. 4249), AKT Antibody (Cat. 9272), Phospho-AKT (Ser473) (D9E) XP Rabbit mAb (Cat. 4060) and Anti-Rabbit IgG HRPlinked antibodies (Cat.7074) were purchased from Cell Signaling Technology (Danvers, USA).

Methanol (HPLC grade) was purchased from Sinopharm Chemical Reagent Co., Ltd. (Shanghai, China). Formic acid (HPLC grade) was purchased from Aladdin Reagent Co., Ltd. (Shanghai, China). The reference standard of shikimic acid (lot No. C11015240) was purchased from Shanghai Macklin Biochemical Co., Ltd. The reference standard of isoengelitin (lot No. ZW190519-14) was purchased from Stanford Analytical Chemicals Inc. The reference standard of 4-dicaffeoylquinic acid (lot No. 34770010) was purchased from ANPEL Laboratory Technologies (Shanghai) Inc. The reference standard of phydroxybenzoic acid (lot No. H1926195) was purchased from Shanghai Aladdin Reagent Co., Ltd. The reference standards of engeletin (lot No. 111906-201102), astilbin (lot No. 111798-201504), ferulic acid (lot No. 110773-201313), luteolin (lot No. 111520-201605), isochlorogenic acid C (lot No. 111894201102), salicylic acid (lot No. 100106-201104), nicotinic acid (lot No. 100434-201603) were purchased from National Institutes for Food and Drug Control (Beijing, China). The reference standards of neoastilbin (lot No. DST191209-077), isoastilbin (lot No. DST190922-216), neoisoastilbin (lot No. DST191025-078) were purchased from Chengdu Pufei De Biotech Co., Ltd.

\subsection{Animals and experimental design}

A total of 40 male Spontaneously hypertensive rats (SHR) (180-220g), aged 10 weeks, were provided by Beijing Vital River Laboratory Animal Technologies Co. Ltd (Beijing, China, certificate number: SCXK-JING 2016-0006). All animals were maintained under standard laboratory conditions with a regular 12-hour light/dark cycle, a relative humidity of $50 \pm 5 \%$, and temperature of $25 \pm 2^{\circ} \mathrm{C}$. The experimental procedures were approved by the Institutional Animal Ethics Committee (IAEC), Guangxi Medical University.

The rats were provided with standard rat chow and water ad libitum. After an acclimatization period for two weeks, the basic value of blood pressure, body weight, and heart rate were measured. SHR rats were randomly assigned to five groups based on the basic value of blood pressure: model group (saline, 10 $\mathrm{mL} / \mathrm{kg}$ ), captopril group (0.03 g/ $\mathrm{kg}$ ), LFG high dose group (20 g/ $\mathrm{kg})$, LFG middle dose group $(10 \mathrm{~g} / \mathrm{kg})$, LFG low dose group ( $5 \mathrm{~g} / \mathrm{kg})$, eight rats per group. Saline served as a negative control and captopril, a proven hypertensive drug [20], as a positive control. LFG and captopril were administered intragastrically twice a day for 3 weeks. The systolic blood pressure, body weight, and heart rate of rats were measured 
every three days. The blood pressure and heart rate were measured using ALC-NIBP non-invasive blood pressure system (Shanghai Alcott Biotech Co., Ltd, China) at the tail artery employing tail-cuff method, while body weight was measured using an electronic balance (CP214, Ohaus, USA). When the experimental period was complete, rats were anaesthetized and blood was collected from the abdominal aorta. The serum samples were obtained after the blood samples were centrifuged. The heart and thoracic aorta were collected respectively for hematoxylin-eosin (H\&E) staining and stored at $-80^{\circ} \mathrm{C}$ for real-time PCR and western blot analysis.

\subsection{Determination of serum NO, ANG $₫$, and ET-1 content}

Serum NO level was measured by Nitric oxide (NO) assay kit obtained from Nanjing Jiancheng

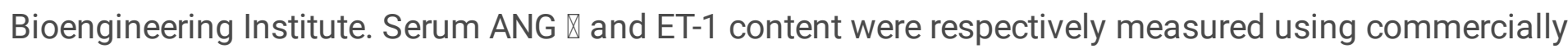
available immunoassay ELISA kits for rats.

\subsection{Heart histopathological analysis}

Heart tissue samples were fixed in $4 \%$ paraformaldehyde, dehydrated, and washed. The samples were embedded in paraffin and sliced into $5 \mu \mathrm{m}$ by paraffin slicing machine. The sections were dewaxed in xylene and dehydrated in descending grades of ethanol. The sections were stained by hematoxylin for 5 min. After washing, the sections were treated with eosin solution for 2 min for coloration, followed by ethanol dehydration, and neutral gum sealing. Finally, the specimens were further observed and photographed under a BX53 microscope (Olympus, Takachiho, Japan).

\subsection{Network pharmacology analysis \\ 2.5.1. Composite compounds of LFG}

LFG consists of three herbs, namely Centella asiatica (L.) Urb., Eclipta prostrata (L.) L., Smilax glabra Roxb. To collect the compounds of LFG, we used the TCM Systems Pharmacology Database [21] (TCMSP, http://tcmspw.com/tcmsp.php) (accessed 18th January 2018), TCM Integrated Database [22] (TCMID, http://www.megabionet.org/tcmid/) (accessed 18th January 2018), and Bioinformatics Analysis Tool for Molecular mechanism of TCM [23] (BATMAN-TCM, http://bionet.ncpsb.org/batman-tcm/) (accessed 19th January 2018). Candidate compounds were screened according to the criteria of oral bioavailability $(\mathrm{OB})>30 \%$ of the pharmacokinetic parameters [24].

\subsubsection{Targets collection}

The SMILES of the candidate compounds in LFG as the inputs of target fishing database were obtained from Pubchem database (https://pubchem.ncbi.nlm.nih.gov/). Subsequently, the predicted targets were retrieved from ChemMapper database [25] (http://lilab.ecust.edu.cn/chemmapper/) (accessed 26th January 2018), an online tool based on 3D similarity for targets prediction. The targets associated with hypertension were collected by Text-mined Hypertension, Obesity and Diabetes candidate gene database [26] (T-HOD, http://bws.iis.sinica.edu.tw/THOD/) (accessed 28th January 2018), a tool developed to help trace existing research on three kinds of cardiovascular disease. Furthermore, the overlapped targets that 
were both related to the candidate compounds and hypertension were kept for network construction and analysis.

\subsubsection{Network construction}

Network construction was performed as follows: (1) Herb-compound network; (2) Compound-target network; (3) Hypertension-target network; (4) The protein-protein interaction (PPI) network of intersection targets between compounds and hypertension targets.

All the networks were created via utilizing Cytoscape (htttps://cytoscape.org/, Version 3.2.1), a general platform for complex network analysis and visualization [27]. The PPI network was constructed by Search Tool for the Retrieval of Interacting Genes/Proteins database [28] (STRING, https://string-db.org/, Version 11.0), and then visualized in Cytoscape. The topological parameters of nodes in PPI network were calculated by Network Analyzer, and the nodes whose degree values were twice the average degree values of the network were selected as the hub targets for further analysis.

\subsubsection{Pathway enrichment}

The Database for Annotation, Visualization and Integrated Discovery [29] (DAVID, https://david.ncifcrf.gov/, Version 6.8) was applied for Kyoto Encyclopedia of Genes and Genomes (KEGG) pathway enrichment analysis of the hub targets. $P$-values were set at 0.05 as the cut-off criterion.

\subsection{Real-time RT-PCR analysis}

Quantitative PCR analysis was performed to examine the mRNA expression levels of predicted hub targets. In brief, total RNA was extracted from thoracic aorta tissue using Trizol reagent, and the quality and purity of RNA were evaluated by Nanodrop2000 (Thermo Fisher, USA). The RNA (2 $\mu$ g) was reversetranscribed using the High Capacity cDNA Reverse Transcription Kit under reaction conditions with $25^{\circ} \mathrm{C}$ for $10 \mathrm{~min}, 37^{\circ} \mathrm{C}$ for $120 \mathrm{~min}$ and $85^{\circ} \mathrm{C}$ for $5 \mathrm{~min}$. Real-time PCR amplification was performed with SYBR Green Master Mix on the 7300 Real time PCR system (Applied Biosystems) following the manufacturer's instructions. The relative RNA expression levels were calculated using the $\Delta \Delta$ cycle threshold method [30], and $\beta$-actin was applied to normalize the expression levels of target genes for each sample. Primer sequences were listed in Table 1. 
Table 1

Primer sequence used for PCR analysis

\begin{tabular}{|c|c|c|}
\hline Gene & Forward & Reverse \\
\hline NOS3 & 5'-CAGAGATTGGCATGAGGGACC-3' & 5'-TCCACAGTGATGAGGTTGTCC-3' \\
\hline SRC & 5'-CAAGATCACTAGACGGGAATCAG-3' & 5'-GTTTCACATTTAGGCCCTTGG-3' \\
\hline PTGS2 & 5'-ATCAGAACCGCATTGCCTCT-3' & 5'-GCCAGCAATCTGTCTGGTGA-3' \\
\hline NGF & 5'-GTCTGGGCCCAATAAAGGCT-3' & 5'-TGTACGCCGATCAAAAACGC-3' \\
\hline VEGFA & 5'-CGTCCTGTGTGCCCCTAATG-3' & 5'-TGTGCTGGCTTTGGTGAGGT-3' \\
\hline EGF & 5'-CTACTACAGGACTCGGAAGCAG-3' & 5'-GTTGGGGACCAGAAGACACC-3' \\
\hline EGFR & 5'-AGCCGTCCTGTCCAACTATG-3' & 5'-TTGCTAAATCGCACAGCACC-3' \\
\hline ERK2 & 5'-ATTGGTCAGGACAAGGGCTCA-3' & 5'-CCACTACGACCAGAACTGCC-3' \\
\hline CCL2 & 5'-TGCAGGTCTCTGTCACGCTTC-3' & 5'-TTCTCCAGCCGACTCATTGG-3' \\
\hline NPY & 5'-GCCAGATACTACTCCGCTCTG-3' & 5'-GTCTCAGGGCTGGATCTCTTG-3' \\
\hline ESR1 & 5'-CAGACAGGGAGCTGGTTCATA-3' & 5'-GCACACTCCAGAAGGTGAACT-3' \\
\hline TH & 5'-GTCTCAGAGCAGGATGCCAAG-3' & 5'-ATCCTCGATGAGACTCTGTCGC-3' \\
\hline$\beta$-actin & 5'-GTCAGGTCATCACTATCGGCAAT-3' & 5'-AGAGGTCTTTACGGATGTCAACGT-3' \\
\hline
\end{tabular}

\subsection{Western blot analysis}

The key protein expression levels of PI3K-AKT signaling pathway were determined by western blot analysis. Total proteins extracted from thoracic aorta by lysis buffer supplemented with phosphatase and protease inhibitors. The protein concentrations were measured by BCA Protein Assay Kit. Protein samples $(30 \mu \mathrm{g})$ were separated on $10 \%$ sodium dodecyl sulphate-polyacrylamide gel electrophoresis (SDS-PAGE) and then transferred to polyvinylidene difluoride (PVDF) membranes. The membranes were blocked for 1 hour at $4^{\circ} \mathrm{C}$ with $5 \%$ nonfat milk, while the phosphorylated proteins were blocked with $5 \%$ bovine serum albumin (BSA), and incubated overnight with anti-PI3K (1:1000) antibody, anti-AKT (1:1000) antibody, anti-p-AKT (1:1000) antibody and anti-GAPDH (1:5000) antibody, respectively. The membranes were washed 3 times and incubated with secondary antibody (horseradish peroxidase-conjugated, 1:5000 dilution) for 1 hour at room temperature. The GAPDH was applied to show equal protein loading for each sample. The bands were visualized by chemiluminescence using MiniChemi 610 Plus (Sage Creation, Beijing), and Image $\mathrm{J}$ software (Version 1.43) was used to analysis the intensity of bands.

\subsection{Molecular docking}

Molecular docking is a computational tool that can predict the interaction energy between receptors and ligands. The binding mode and affinity between targets and compounds was performed using the Surflex-Dock (SFXC) module of Sybyl X2.0 and visualized with PyMOL (Version 1.5.0.3). The structures of 
the potential active compounds related to the potential targets were downloaded from the Pubchem database, and converted into mol2 format by Open Babel GUI (Version 2.2.1). All the crystal structures of the targets were downloaded from Protein Data Bank (PDB, https://www.rcsb.org/), including endothelial nitric oxide synthase (NOS3) (PDB ID: 4D10), SRC (PDB ID: 1A07), PI3K (PDB ID: 4L23), and AKT (PDB ID: 1H10). Before docking, the proteins were subjected to the necessary preparation steps (remove water molecules, and add hydrogen atoms), and ligands were optimized by Tripos force field. The total score (pKd) calculated by SFXC was used to determine the affinity of receptors and ligands. The total score greater than 6 was regarded as good protein-ligand binding.

\subsection{UPLC-QTOF/MS analysis of LFG}

LFG extract was dissolved by ultrasonification in $50 \%$ methanol (in water) for 1 hour, and filtered through a $0.22 \mu \mathrm{m}$ syringe filter before analysis.

UPLC analysis conditions were as follows: Separation of the compounds was carried out on a Dionex Ultimate 3000 UPLC system (Thermo fisher, USA) equipped with a SunFire C18 column ( $250 \mathrm{~mm} \times 4.6$ $\mathrm{mm}$, i.d., $4.6 \mu \mathrm{m}$, Waters, USA). The analytical column was maintained at a temperature of $35^{\circ} \mathrm{C}$ and the mobile phase was composed of water $A$ (containing $0.1 \%$ formic acid) and acetonitrile $B$. A solvent gradient system was used: $10-10 \%$ B for $0-10 \mathrm{~min}, 10-30 \%$ B for $10-50 \mathrm{~min}, 30-90 \%$ B for $50-58 \mathrm{~min}$, $90-90 \%$ B for $58-64 \mathrm{~min}, 90-10 \%$ B for $64-65 \mathrm{~min}$ and $10-10 \% \mathrm{~B}$ for $65-75 \mathrm{~min}$. The flow rate was 0.8 $\mathrm{ml} / \mathrm{min}$. The injection volume was $10 \mu \mathrm{l}$. A DAD detector and a mass spectrometer detector were connected in series behind the column. The outlet of the DAD detector was split into the mass spectrometer detector with a split ratio of 4:1.

MS analysis conditions were as follows: MS analysis was performed on the Bruker definition accurate mass quadrupole time-of-flight (Q-TOF) Impact II mass spectrometer (Bruker Daltonics Inc., GER) equipped with electrospray ionization (ESI) source. The capillary voltage was set at $2500 \mathrm{~V}$, the dry temperature was $200^{\circ} \mathrm{C}$, the dry gas rate was set at $4.0 \mathrm{~L} / \mathrm{min}$, the energies for collision induced dissociation (CID) were $10 \mathrm{eV}$ for the precursor ion and 32.5-97.5 eV for fragmentation information. A full-scan mass range of $\mathrm{m} / \mathrm{z}$ 50-2000 was scanned. Data were acquired in Line Spectra mode.

\subsection{Statistical analysis}

Statistical analysis was performed by SPSS software (Version 22.0). The data were expressed as mean \pm SEM. The parameters among different groups were compared by one-way analysis of variance (ANOVA) followed by LSD (equal variances) or Dunnett's T3 (unequal variances). $P$-value $<0.05$ was considered as statistically significant.

\section{Results}

\subsection{LFG exerts an anti-hypertensive effect in SHR}


To assess the anti-hypertensive effect of LFG, SHR were treated with different doses of LFG for 3 weeks. As shown in Fig. 2A, the blood pressure of LFG treatment groups was significantly decreased compared to model group $(P<0.05)$. But there were no differences both in body weight and heart rate between LFG treatment groups and model group $(P>0.05)$ (Fig. 2B-C).

\subsection{Effect of LFG on serum NO, ANG $\triangle$ and ET-1 levels}

Serum NO, ANG $\triangle$ and ET-1 levels of animals from various groups were summarized in Fig. 3 . The level of serum NO increased significantly in LFG high-dose group and LFG middle-dose group compared to model group ( $P<0.01$ or 0.05 ) (Fig. 3A). However, there was no significant difference of LFG low-dose group compared to model group, and significant differences in the ANG $\otimes$ and ET-1 contents between model group and treatment groups were not observed $(P>0.05)$ (Fig. 3A-C).

\subsection{Effect of LFG on myocardial morphological change}

As shown in Fig. 4, myocardial samples from model group developed obvious cardiac hypertrophy and fibrosis, as the swelling and sparsely arranged myocardial cells, increased single myocardial cell area, granular content, and karyopyknosis in some myocardial cells (Fig. 4A). These phenomena were improved in captopril group (Fig. 4B). Compared with model group, LFG treatment groups reduced the swelling degree of myocardial cells and decreased the single myocardial cell area, and three doses of LFG administration decreased the area of myocardial fibrosis to some extent (Fig. 4C-E).

\subsection{Composite compounds of LFG}

A total of 70 compounds were collected according to the ADME threshold of $O B \geq 30 \%$, and the herbcompound network was shown in Fig. 5A, which included 73 nodes and 77 edges. The ellipse nodes represented herbs, and the hexagon nodes were compounds. Among these compounds, yellow parts were the common components of Centella asiatica (L.) Urb. and Smilax glabra Roxb., and quercetin (red hexagon) was common compounds of 3 herbs.

\subsection{Compound-target network}

We predicted the putative targets of compounds based on the similarities in drug structures by ChemMapper database. While 17 herbal compounds' relevant targets were not observed, so they were removed from the list of 70 herbal compounds. A total of 765 targets were predicted to be hit by the chemical constituents containing in LFG, and the detailed information about the targets were shown in our previous study [19]. The compound-target network was shown in Fig. 5B, the $V$ shape nodes represented 53 compounds, and the 765 yellow nodes were targets corresponding to the components.

\subsection{Hypertension targets and key targets}


After collection, 828 hypertension related genes were obtained from T-HOD database (Additional file 1, Table S1). The hypertension-target network was established, as shown in Fig. 5C, the red node in the center was hypertension, and the green nodes represented gene targets. A total of 101 overlapped genes, which were both related to candidate compounds and hypertension were kept for PPI network construction using STRING database. The PPI network was visualized by Cytoscape software, as shown in Fig. 5D, the size of nodes changes from big to small according to degree values, and the 12 yellow nodes were recognized as the key targets after screening, including NOS3, SRC, prostaglandin G/H synthase 2 (PTGS2), nerve growth factor (NGF), vascular endothelial growth factor A (VEGFA), epidermal growth factor (EGF), epidermal growth factor receptor (EGFR), mitogen-activated protein kinase 1 (ERK2), C-C motif chemokine 2 (CCL2), neuropeptide Y (NPY), estrogen receptor (ESR1), and tyrosine 3monooxygenase $(\mathrm{TH})$. There were 37 compounds associated with these 12 key genes (Table 2). 
Table 2

The information of 12 key targets between compounds and hypertension targets

\begin{tabular}{|c|c|c|c|}
\hline Gene & Compounds & Gene & Compounds \\
\hline VEGFA & glucoside & MAPK1 & Cedar acid \\
\hline NPY & Ferulic Acid & MAPK1 & Ferulic Acid \\
\hline EGF & salicylic acid & Nos3 & L-Bornyl acetate \\
\hline EGF & nicotinic acid & NOS3 & p-Hydroxybenzoic acid \\
\hline EGF & p-Hydroxybenzoic acid & NOS3 & salicylic acid \\
\hline EGFR & naringenin & NOS3 & Pulegone \\
\hline TH & $\begin{array}{l}\text { (1R,2R,5S)-2,6,6-trimethylnorpinan-3- } \\
\text { one }\end{array}$ & NOS3 & Cedar acid \\
\hline TH & Ferulic Acid & NOS3 & nicotinic acid \\
\hline TH & Histidine & NOS3 & Histidine \\
\hline NGF & (-)-alpha-Pinene & NOS3 & (R)-linalool \\
\hline NGF & p-Hydroxybenzoic acid & NOS3 & 5-Hydroxymethylfurfural \\
\hline NGF & (-)-nopinene & ESR1 & L-Bornyl acetate \\
\hline SRC & Shikimic Acid & ESR1 & $\begin{array}{l}(1 \mathrm{R}, 2 \mathrm{R}, 5 \mathrm{~S})-2,6,6 \text {-trimethylnorpinan- } \\
\text { 3-one }\end{array}$ \\
\hline SRC & nicotinic acid & ESR1 & Pratensein \\
\hline SRC & Histidine & ESR1 & alpha-Terthienyl methyl acetate \\
\hline PTGS2 & naringenin & ESR1 & diosgenin \\
\hline PTGS2 & Histidine & ESR1 & (+)-Isoborneol \\
\hline PTGS2 & demethylwedelolactone & ESR1 & sitosterol \\
\hline PTGS2 & acacetin & ESR1 & Stigmasterol \\
\hline PTGS2 & Ferulic Acid & ESR1 & butin \\
\hline PTGS2 & Xanthanoic acid & ESR1 & Baimuxinol \\
\hline PTGS2 & 5-Hydroxymethylfurfural & ESR1 & $\begin{array}{l}\text { (Z)-1-(2,4-dihydroxyphenyl)-3- } \\
\text { (3,4-dihydroxyphenyl)prop-2-en-1- } \\
\text { one }\end{array}$ \\
\hline PTGS2 & (L)-alpha-Terpineol & ESR1 & (-)-nopinene \\
\hline
\end{tabular}




\begin{tabular}{|llll|}
\hline Gene & Compounds & Gene & Compounds \\
\hline PTGS2 & $\begin{array}{l}\text { (1R,2R,5S)-2,6,6-trimethylnorpinan-3- } \\
\text { one }\end{array}$ & ESR1 & 1,8-cineole \\
\hline PTGS2 & Pulegone & ESR1 & (-)-alpha-Pinene \\
\hline PTGS2 & MENTHOL & ESR1 & demethylwedelolactone \\
\hline PTGS2 & L-Limonen & ESR1 & 3'-O-Methylorobol \\
\hline PTGS2 & salicylic acid & ESR1 & wedelolactone \\
\hline PTGS2 & nicotinic acid & ESR1 & luteolin \\
\hline PTGS2 & p-Hydroxybenzoic acid & ESR1 & acacetin \\
\hline PTGS2 & L-Bornyl acetate & ESR1 & Dihydroresveratrol \\
\hline PTGS2 & Cedar acid & ESR1 & naringenin \\
\hline CCL2 & Ferulic Acid & ESR1 & cis-resveratrol \\
\hline MAPK1 & p-Hydroxybenzoic acid & & \\
\hline 3 & & & \\
\hline
\end{tabular}

\subsection{Pathway enrichment of key targets}

To elucidate the crucial pathways among the key targets in hypertension treatment, KEGG pathway enrichment analysis was performed. The top 10 pathways were shown in Fig. 6A, including Bladder cancer, VEGF signaling pathway, Rap1 signaling pathway, Estrogen signaling pathway, HIF-1 signaling pathway, Oxytocin signaling pathway, PI3K-AKT signaling pathway, Pancreatic cancer, Proteoglycans in cancer, and Prolactin signaling pathway. The compound-target-pathway network was summarized in Fig. 6B, where compounds were indicated by purple nodes; gene targets were shown as yellow nodes; 34 pathways were labeled with green nodes.

\subsection{RT-qPCR verification}

To further validate the 12 hub targets predicted from network pharmacology approach, the mRNA expression levels were measured by real-time RT-PCR. As shown in Fig. 7, the results revealed that the mRNA expression levels of NOS3 and SRC in LFG high-dose group increased significantly compared with model group ( $P<0.01$ or 0.05 ). Compared to model group, the mRNA expression level of NOS3 was significantly upregulated in LFG middle-dose group $(P<0.05)$. For the other predicted genes, there were no significant difference between model groups and LFG treatment groups $(P>0.05)$ (Fig. 7C-L). Therefore, we only focused on NOS3 and SRC in the following study.

\subsection{Western blot verification}


The results of RT-qPCR revealed that LFG could regulate the expression of NOS3 and SRC which associated with 10 compounds. At the same time, pathway enrichment analysis of these 2 genes was performed, and the result shown that the antihypertensive effect of LFG may be exerted through PI3KAKT signaling pathway. Then we conducted the compound-target-pathway network of significant genes. As shown in Fig. 8A, compounds were indicated by diamond nodes ( $\mathrm{p}$-Hydroxybenzoic acid, Nicotinic acid, Salicylic acid, (R)-Linalool, Cedar acid, L-Bornyl acetate, Pulegone, 5-Hydroxymethylfurfural, Shikimic acid, and Histidine), and pathways were shown as hexagon nodes.

To further verify the role of PI3K-AKT signaling pathway on the antihypertensive effect of LFG, the protein expression levels of PI3K, AKT, and p-AKT in the thoracic aorta tissue were measured by western blot analysis. As shown in Fig. 8B, expression levels of PI3K and p-AKT were increased in LFG high-dose group and LFG middle-dose group compared to model group ( $P<0.01$ or 0.05$)$. There was no significant difference in the level of total AKT expression between model group and treatment groups $(P>0.05)$.

\subsection{Molecular docking analysis}

To elucidate the interaction between targets (NOS3, SRC, PI3K, and AKT) and the 10 potential active compounds, a molecular docking simulation was performed using Sybyl X2.0 to investigate the binding modes of them. Docking score greater than 6 indicates good protein-ligand binding activity. The docking results of protein-ligand interaction were summarized in Table S2-S5 (Additional file 1). The binding modes of receptors and ligands with good affinity were shown in Fig. 9. In summary, histidine, cedar acid, linalool, and p-hydroxybenzoic acid were successfully docked in NOS3 (Fig. 9A-D). Shikimic acid, salicylic acid, histidine, cedar acid, nicotinic acid, and p-hydroxybenzoic acid were docked in SRC (Fig. 9E-J). Cedar acid, p-hydroxybenzoic acid, and linalool were docked in PI3K (Fig. 9K-M). Cedar acid, shikimic acid, and nicotinic acid were docked in AKT (Fig. 9N-P). Interestingly, cedar acid can be docked in NOS3, SRC, PI3K, and AKT, and p-hydroxybenzoic acid can be docked in NOS3, SRC, and PI3K. Molecular docking results revealed that 7 compounds (Cedar acid, p-hydroxybenzoic acid, histidine, shikimic acid, salicylic acid, linalool, nicotinic acid) exhibited good interaction with NOS3, SRC, PI3K, and AKT.

\subsection{LC-MS/MS analysis of LFG}

In order to verify whether the 7 components predicted by molecular docking exist in LFG, LC-MS/MS was performed to analysis the chemical constituents of LFG. The representative base peak intensity (BPI) chromatogram in negative ion mode was shown in Fig. 10. After comparing the components with the reference standard and LFG (Additional file 2, Figure S1-S18), eighteen compounds were detected, and the detail information of these compounds was shown in Table 3. Among these compounds, four compounds ( $p$-hydroxybenzoic acid, shikimic acid, salicylic acid, nicotinic acid) well docked in NOS3, $\mathrm{SRC}, \mathrm{PI} 3 \mathrm{~K}$, and AKT were confirmed to exist in LFG. 
Table 3

Identify of the compounds of LFG by UPLC- Q-TOF/MS analysis in negative ion mode

\begin{tabular}{|c|c|c|c|c|c|c|c|}
\hline NO. & R-T & $\mathrm{m} / \mathrm{z}$ & MS/MS & $\begin{array}{l}\text { Elemental } \\
\text { composition }\end{array}$ & $\begin{array}{l}\text { lon } \\
\text { form }\end{array}$ & ppm & Compounds \\
\hline 1 & 3.73 & 173.0458 & 93.0354 & $\mathrm{C}_{7} \mathrm{H}_{10} \mathrm{O}_{5}$ & $\begin{array}{l}{[\mathrm{M}-} \\
\mathrm{H}]^{-}\end{array}$ & -1.3 & Shikimic acid \\
\hline 2 & 3.90 & 124.0394 & $78.0334 / 80.0490$ & $\mathrm{C}_{6} \mathrm{H}_{5} \mathrm{NO}_{2}$ & $\begin{array}{l}{[\mathrm{M}-} \\
\mathrm{H}]^{+}\end{array}$ & -0.7 & Nicotinic acid \\
\hline 3 & 18.59 & 137.0244 & 93.0354 & $\mathrm{C}_{7} \mathrm{H}_{6} \mathrm{O}_{3}$ & $\begin{array}{l}{[\mathrm{M}-} \\
\mathrm{H}]^{-}\end{array}$ & 0.2 & $\begin{array}{l}\text { p- } \\
\text { Hydroxybenzoic } \\
\text { acid }\end{array}$ \\
\hline 4 & 19.72 & 353.0882 & 191.0565 & $\mathrm{C}_{16} \mathrm{H}_{18} \mathrm{O}_{9}$ & $\begin{array}{l}{[\mathrm{M}-} \\
\mathrm{H}]^{-}\end{array}$ & -1.0 & $\begin{array}{l}\text { Chlorogenic } \\
\text { acid }\end{array}$ \\
\hline 5 & 21.78 & 353.0881 & $191.0563 / 173.0456$ & $\mathrm{C}_{16} \mathrm{H}_{18} \mathrm{O}_{9}$ & $\begin{array}{l}{[\mathrm{M}-} \\
\mathrm{H}]^{-}\end{array}$ & -0.9 & $\begin{array}{l}\text { 4- } \\
\text { Dicaffeoylquinic } \\
\text { acid }\end{array}$ \\
\hline 6 & 28.69 & 515.1196 & $191.0563 / 179.0351$ & $\mathrm{C}_{25} \mathrm{H}_{24} \mathrm{O}_{12}$ & $\begin{array}{l}{[\mathrm{M}-} \\
\mathrm{H}]^{-}\end{array}$ & -0.1 & $\begin{array}{l}\text { Isochlorogenic } \\
\text { acid C }\end{array}$ \\
\hline 7 & 35.37 & 193.0509 & $106.0438 / 134.0376$ & $\mathrm{C}_{10} \mathrm{H}_{10} \mathrm{O}_{4}$ & $\begin{array}{l}{[\mathrm{M}-} \\
\mathrm{H}]^{-}\end{array}$ & -1.4 & Ferulic acid \\
\hline 8 & 36.84 & 449.1089 & $151.0036 / 285.0405$ & $\mathrm{C}_{21} \mathrm{H}_{22} \mathrm{O}_{11}$ & $\begin{array}{l}{[\mathrm{M}-} \\
\mathrm{H}]^{-}\end{array}$ & 0.1 & Neoastilbin \\
\hline 9 & 38.09 & 449.1099 & $151.0040 / 285.0413$ & $\mathrm{C}_{21} \mathrm{H}_{22} \mathrm{O}_{11}$ & $\begin{array}{l}{[\mathrm{M}-} \\
\mathrm{H}]^{-}\end{array}$ & -2.3 & Astilbin \\
\hline 10 & 40.67 & 449.1095 & $151.0037 / 285.0410$ & $\mathrm{C}_{21} \mathrm{H}_{22} \mathrm{O}_{11}$ & $\begin{array}{l}{[\mathrm{M}-} \\
\mathrm{H}]^{-}\end{array}$ & -1.3 & Neoisoastilbin \\
\hline 11 & 41.50 & 449.1094 & $151.0037 / 285.0408$ & $\mathrm{C}_{21} \mathrm{H}_{22} \mathrm{O}_{11}$ & $\begin{array}{l}{[\mathrm{M}-} \\
\mathrm{H}^{-}\end{array}$ & -0.9 & Isoastilbin \\
\hline 12 & 43.45 & 433.1147 & $180.0068 / 269.0463$ & $\mathrm{C}_{21} \mathrm{H}_{22} \mathrm{O}_{10}$ & $\begin{array}{l}{[\mathrm{M}-} \\
\mathrm{H}]^{-}\end{array}$ & -1.6 & Engeletin \\
\hline 13 & 47.33 & 433.1137 & $180.0059 / 269.0448$ & $\mathrm{C}_{21} \mathrm{H}_{22} \mathrm{O}_{10}$ & $\begin{array}{l}{[\mathrm{M}-} \\
\mathrm{H}]^{-}\end{array}$ & -0.4 & Isoengelitin \\
\hline 14 & 49.31 & 137.0245 & 93.0354 & $\mathrm{C}_{7} \mathrm{H}_{6} \mathrm{O}_{3}$ & $\begin{array}{l}{[\mathrm{M}-} \\
\mathrm{H}^{-}\end{array}$ & -0.5 & Salicylic acid \\
\hline 15 & 55.45 & 285.0409 & $133.0297 / 151.0033$ & $\mathrm{C}_{15} \mathrm{H}_{10} \mathrm{O}_{6}$ & $\begin{array}{l}{[\mathrm{M}-} \\
\mathrm{H}]^{-}\end{array}$ & -1.7 & Luteolin \\
\hline
\end{tabular}




\begin{tabular}{|c|c|c|c|c|c|c|c|}
\hline NO. & R-T & $\mathrm{m} / \mathrm{z}$ & MS/MS & $\begin{array}{l}\text { Elemental } \\
\text { composition }\end{array}$ & $\begin{array}{l}\text { lon } \\
\text { form }\end{array}$ & ppm & Compounds \\
\hline 16 & 56.15 & 313.0368 & 298.0126 & $\mathrm{C}_{16} \mathrm{H}_{10} \mathrm{O}_{7}$ & $\begin{array}{l}{[\mathrm{M}-} \\
\mathrm{H}]^{-}\end{array}$ & -4.4 & Wedelolactone \\
\hline 17 & 59.00 & 503.3381 & $389.2861 / 437.3066$ & $\mathrm{C}_{30} \mathrm{H}_{48} \mathrm{O}_{6}$ & $\begin{array}{l}{[\mathrm{M}-} \\
\mathrm{H}]^{-}\end{array}$ & -0.6 & $\begin{array}{l}\text { Madecassic- } \\
\text { acid }\end{array}$ \\
\hline 18 & 60.20 & 487.3431 & $379.3034 / 409.3156$ & $\mathrm{C}_{30} \mathrm{H}_{48} \mathrm{O}_{5}$ & $\begin{array}{l}{[\mathrm{M}-} \\
\mathrm{H}]^{-}\end{array}$ & -0.4 & Asiatic acid \\
\hline
\end{tabular}

\section{Discussion}

Hypertension is a major risk factor for cardiovascular and cerebrovascular diseases as current complications such as stroke, heart failure, myocardial infarction and renal failure [31]. Blood pressure can be controlled by changing diet and lifestyle, but drug treatment is used when these methods are not effective. Drugs used to treat hypertension include calcium channel blockers, $\beta$-blockers, angiotensin converting enzyme inhibitors (ACEI) and angiotensin II receptor blockers. However, various side effects of these drugs have been reported [32,33]. Long-established Chinese herbal formulas can not only stabilize the blood pressure, but also improve the quality of life, minimize the risk factors associated with high blood pressure, and prevent organ damage to improve patient survival [34]. In this study, we used a SHR model to access the effects of LFG on attenuating blood pressure. Our data showed that compared with model group, LFG significantly reduced blood pressure and increased serum NO content in SHR rats. LFG ameliorated the pathological changes such as cardiac hypertrophy and interstitial inflammation.

Although the animal experimental results showed that LFG could exert an antihypertensive effect in SHR, the active compounds and underlying molecular mechanisms are not clear. In the present study, a network pharmacology approach was applied to identify the active compounds, corresponding targets, and pharmacological mechanisms of LFG antihypertensive effect. Based on the results of public databases searching, 53 candidate compounds from LFG were collected, which linked to 765 potential targets. 828 hypertension associated targets were retrieved. The potential targets of antihypertensive effect were found by comparing LFG genes with hypertension genes, and 12 key targets were obtained after screening. After pathway enrichment of 12 key targets, 37 components and 34 pathways were found, among which the top 10 pathways were Bladder cancer, VEGF signaling pathway, Rap1 signaling pathway, Estrogen signaling pathway, HIF-1 signaling pathway, Oxytocin signaling pathway, PI3K-AKT signaling pathway, Pancreatic cancer, Proteoglycans in cancer, and Prolactin signaling pathway were screened.

Twelve key genes were validated by RT-qPCR, and the results showed that LFG could upregulate the mRNA expression of NOS and SRC in thoracic aorta. Endothelial nitric oxide synthase 3 (NOS3) is mainly expressed in endothelial cells. NO produced by NOS3 is a major vascular protective agent because of its vasodilator, antioxidant and antiproliferative properties [35]. It was reported that demethylasterriquinone 
b1 induced Akt activation may stimulate the expression and activity of NOS3 and inhibit the expression of p22phox subunit of NADPH oxidase to reduce oxidative stress and subsequently improve vascular endothelial dysfunction [36]. It was showed that AVE3085 can effectively reduce the blood pressure of hypertensive rats. AVE3085 restored the impaired endothelium dependent relaxations in the aortae of SHRs, so the improvement of endothelial function may be related to its antihypertensive effect [37]. SRC tyrosine protein kinase is a non-receptor protein tyrosine kinase. It plays an important role in regulation of cell differentiation, proliferation, and transformation, and also plays an important biological role in diseases such as hypertension and tumor $[38,39]$. It was reported that SRC was involved in the production of NO and prostaglini2 (PGI2) induced by VEGF, suggesting that SRC was involved in the process of reducing vascular tension, relaxing blood vessel, and lowering blood pressure of VEGF in the vascular endothelium [40]. Taken together, we demonstrated that the expression of NOS3 and SRC were altered in model group, and LFG attenuated hypertension through restoring their expression to normal levels.

Pathway enrichment results showed that PI3K-AKT signaling pathway was closely related to the expression of NOS3 and SRC. To verify this result, western blot was used to detect the key proteins (PI3K, $A K T$, and p-AKT) of PI3K-AKT signaling pathway. The results showed that LFG significantly increased the protein expression levels of PI3K and p-AKT in SHR rats, suggesting that LFG may play a role through the PI3K-AKT signaling pathway. The PI3K-AKT signaling pathway is activated by many types of cellular stimuli or toxic insults and regulates fundamental cellular functions such as transcription, translation, proliferation, growth, and survival. PI3K catalyzes the production of phosphatidylinositol-3,4,5triphosphate (PIP3) at the cell membrane. PIP3 in turn serves as a second messenger that helps to activate AKT. Once activated, AKT can control key cellular processes by phosphorylating substrates involved in apoptosis, protein synthesis, metabolism, and cell cycle. It was reported that the AKT activity of blood vessels characterized by Ser 473 phosphorylation is downregulated in a number of hypertensive rat models, including in SHRs [41]. Importantly, phosphorylation of AKT at Ser473 contributes to NOS3 activation [42]. SRC regulates the activity of PI3K-AKT signaling, and contributes to improving endothelial dysfunction [43]. Taken together, the mechanism of LFG exerting antihypertensive effect was showed in Fig. 11. LFG can reduce the blood pressure of SHR rats, which might be attributed to upregulating SRC expression level, activating of PI3K-AKT-NOS3 signaling pathway to further increase the serum NO level for promoting vasodilation.

Molecular docking study further supported that p-hydroxybenzoic acid, cedar acid, shikimic acid, salicylic acid, nicotinic acid, linalool, and histidine can be well binding with NOS3, SRC, PI3K, and AKT. These compounds were potential active ingredients for LFG antihypertensive effects. p-Hydroxybenzoic acid can be isolated from a variety of plants, such as oil palm, Vitex negundo and virgin olive oil [44-46]. It was reported that p-hydroxybenzoic acid possessed a variety of biological properties, including antiinflammatory, antioxidant cardio protective and vasodilatory effects [47]. p-Hydroxybenzoic acid caused a decrease in blood pressure and dilatation of the aortic rings. The biological principles were to act directly on the vascular smooth muscle to cause vasodilatation, and indirectly by stimulating the release of NO from the vascular endothelium to promote the vasodilator activity of the compound [48]. Cedar 
acid, one of the phenolic compounds, is abundantly present in olives, dates, spices, pumpkin, grapes, acai palm and other plants [49]. Also, it exhibits multi-pharmacological properties such as antihypertensive, antioxidant, anti-inflammatory, and hepatoprotective activities [50,51]. Cedar acid showed antihypertensive activity in hypertensive rats induced by N-nitro-L-arginine methyl ester (L-NAME). It was evidenced that Cedar acid treatment might reduce the blood pressure, lipid peroxides, and increase NO availability and antioxidant levels in blood samples of rats [52]. Shikimic acid originally extracted from Chinese star anise, exerts diverse pharmacological actions such as analgesic, anti-inflammatory, and antioxidant effects [53-55]. A previous study indicated that Shikimic acid inhibited inflammatory events including the production of NO and the expression of pro-inflammatory cytokines [56]. Chemically, Salicylic acid is 2-hydroxybenzoic acid or orthohydrobenzoic acid. Sources of Salicylic acid include willow bark, sweet birch, and wintergreen leaves, and it can also be synthesized artificially [57]. Salicylic acid, the Xinjiang red raspberry fruit extract, moderately reduced SHR blood pressure, the effect may be related to increasing serum NO, SOD activity and T-AOC levels, enhanced NOS3 mRNA expression [58]. Niacin, also known as vitamin B3, illustrates the many functions of water-soluble vitamins. Niacin has been widely used in the treatment of dyslipidemia and atherosclerotic coronary heart disease [59]. In addition to its lipid-lowering effect, Niacin also has powerful antioxidant and anti-inflammatory properties [60]. Cho etc. showed that long-term Niacin administration improves hypertension and proteinuria, and attenuates the accumulation of lipids in the remnant kidneys of animals with CRF induced by subtotal nephrectomy [61]. Linalool is a monoterpene and is generally considered to be the main component of essential oils obtained from aromatic plant species [62]. Linalool showed a wide range of biological activities including anti-inflammatory, anti-cancer, anti-hyperlipidemic, anti-microbial effects [63]. A data showed that sub-chronic treatment with Linalool reduced the development of hypertension in SHR, reducing cardiac hypertrophy, increasing IL-10 anti-inflammatory cytokine, improving vasodilatory function and decreasing vasoconstriction [64]. Histidine is an essential amino acid in humans and other mammals which exhibits antioxidant and anti-inflammatory properties [65]. In a dietary intervention and evaluation experiment, the relationship between the intake of specific amino acids and blood pressure levels was analyzed, and the results demonstrated that intakes of Methionine and Alanine were associated positively with higher blood pressure, while intakes of Threonine and Histidine had inverse associations [66]. Toba etc. have demonstrated the antihypertensive effect of L-histidine after oral administration in SHR. These effects may be mediated by central histamine H3 receptors and appear to be related to an increase in NO in the rostral ventrolateral medulla [67].

After comparing the components with the reference standard and LFG by LC-MS/MS, p-hydroxybenzoic acid, shikimic acid, salicylic acid, and nicotinic acid were confirmed to exist in LFG. In conclusion, phydroxybenzoic acid, shikimic acid, salicylic acid, and nicotinic acid might be the underlying compounds of LFG to play an antihypertensive effect.

\section{Conclusions}

LFG can reduce the blood pressure of SHR rats, which might be attributed to upregulating SRC expression level, activating of PI3K-AKT-NOS3 signaling pathway to further increase the serum NO level for 
promoting vasodilation. $\mathrm{p}$-Hydroxybenzoic acid, shikimic acid, salicylic acid, and nicotinic acid might be the underlying active compounds which affects the potential targets for LFG antihypertensive effect.

\section{Declarations}

\section{Acknowledgements}

Not applicable

\section{Authors' contributions}

HG, DZ, ZS contributed to designing the work. QL, TL and WC wrote the manuscript, analyzed and interpreted of data. QL, TL, SH, XL, WZ, QZ, XC, SG and YH performed the experiments. HG and DZ provided reagents/materials/analysis tools. All authors read and approved the final version.

\section{Funding}

This work was supported by Guangxi Natural Science Foundation (2020GXNSFAA259030, 2018GXNSFAA138096); Guangxi Science and Technology Projects (GUIKEGONG 1598013-8); Open Project of Guangxi Key Laboratory of Regenerative Medicine (GUIZAIZHONGKAI 201903), Guangxi Firstclass Discipline Project for Pharmaceutical Sciences (GXFCDP-PS-2018); Natural Science Foundation of Guangxi University of Chinese Medicine (2019QN004).

\section{Competing interests}

The authors declare that they have no competing interests.

\section{Availability of data and materials}

The datasets used and/or analyzed during the current study are available from the corresponding author on reasonable request.

\section{Consent for publication}

Not applicable.

\section{Ethics approval and consent to participate}

The experimental procedures were approved by the Institutional Animal Ethics Committee (IAEC), Guangxi Medical University.

\section{Supplementary information}

Additional file 1: Table S1. The 828 significant genes associated with hypertension. Table S2. Docking results of 10 active ingredients and NOS3. Table S3. Docking results of 10 active ingredients and SRC. 
Table S4. Docking results of 10 active ingredients and PI3K. Table S5. Docking results of 10 active ingredients and AKT.

Additional file 2: Figure S1-S18. The comparing of components with the reference standard and LFG.

\section{Abbreviations}

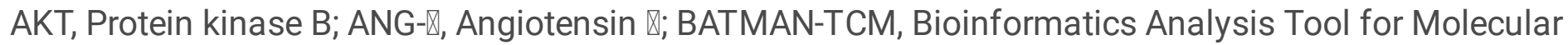
Mechanism of Traditional Chinese Medicine; BCA, Bicinchoninic acid; BSA, Bovine Serum Albumin; CCL2, C-C motif chemokine 2; DAVID, Database for Annotation, Visualization and Integrated Discovery; EGF, Epidermal growth factor; EGFR, Epidermal growth factor receptor; ESR1, Estrogen receptor; ET-1, Endothelin 1; H\&E staining, Hematoxylin-eosin staining; KEGG, Kyoto Encyclopedia of Genes and Genomes; LFG, Lei-gong-gen formula granule; ERK2, Mitogen-activated protein kinase 1; NGF, Nerve growth factor; NO, Nitric oxide; NOS3, Endothelial nitric oxide synthase; NPY, neuropeptide Y; OB, Oral bioavailability; PDB, Protein Data Bank; PI3K, Phosphatidylinositol 3 kinase; PTGS2, Prostaglandin G/H synthase 2; SHR, Spontaneously hypertensive rats; SRC, Proto-oncogene tyrosine-protein kinase SRC; STRING, Search Tool for the Retrieval of Interacting Genes/Proteins; TCMID, Traditional Chinese Medicines Integrated Database; TCMSP, Traditional Chinese Medicine Systems Pharmacology Database and Analysis Platform; TH, Tyrosine 3-monooxygenase; T-HOD, Text-mined Hypertension, Obesity and Diabetes candidate gene database; UPLC-QTOF/MS, Ultra performance liquid chromatography/quadrupole time-of-flight mass spectrometry; VEGFA, Vascular endothelial growth factor A.

\section{References}

1. Wang H, Liu C, Zhai J, Shang H. Niuhuang Jiangya Preparation (a traditional Chinese patent medicine) for essential hypertension: A systematic review. Complement Ther Med. 2017;31:90-9.

2. Li Y, Yang L, Wang L, Zhang M, Huang Z, Deng Q, et al. Burden of hypertension in China: A nationally representative survey of 174,621 adults. Int J Cardiol. 2017;227:516-23.

3. Wolf-Maier K, Cooper RS, Banegas JR, Giampaoli S, Hense HW, Joffres M, et al. Hypertension prevalence and blood pressure levels in 6 European countries, Canada, and the United States. JAMA. 2003;289(18):2363-9.

4. Xiong X, Wang P, Li X, Zhang Y. Shenqi pill, a traditional Chinese herbal formula, for the treatment of hypertension: A systematic review. Complement Ther Med. 2015;23(3):484-93.

5. Wu W, Yang S, Liu P, Yin L, Gong Q, Zhu W. Systems Pharmacology-Based Strategy to Investigate Pharmacological Mechanisms of Radix Puerariae for Treatment of Hypertension. Front Pharmacol. 2020;11:345.

6. Ye XT, Zhang BX, Wang HH, He SB, Zhang XH, Wang Y. Study on mechanism for anti-hypertension efficacy of Eucommiae Cortex through assistant analysis systems for acting mechanisms of traditional Chinese medicine. China Journal of Chinese Materia Medica. 2015;40(19):3718-22. 
7. Lan TJ, Liang WP, Feng QY, Yun CX, Guo HW. Multi-index integrated evaluation method optimizes water-extraction of Leigong Antihypertensive Granules. Journal of Chinese Medicinal Materials. 2018(9):2171-4.

8. Xin RB, Meng GY, He L, Lu J, Huang WY, Ye LL, et al. Sampling survey of hypertension in Zhuang nationality in Tiandong county. Guangxi Medical Journal. 2011;33(8):963-5.

9. Razali NNM, Ng CT, Fong LY. Cardiovascular Protective Effects of Centella asiatica and Its Triterpenes: A Review. Planta Med. 2019;85(16):1203-15.

10. Bunbupha S, Prachaney P, Kukongviriyapan U, Kukongviriyapan V, Welbat JU, Pakdeechote P. Asiatic acid alleviates cardiovascular remodelling in rats with L-NAME-induced hypertension. Clin Exp Pharmacol Physiol. 2015;42(11):1189-97.

11. Liao MY, Chuang CY, Hsieh MJ, Chou YE, Lin CW, Chen WR, et al. Antimetastatic effects of Eclipta prostrata extract on oral cancer cells. Environ Toxicol. 2018;33(9):923-30.

12. Chung IM, Rajakumar G, Lee JH, Kim SH, Thiruvengadam M. Ethnopharmacological uses, phytochemistry, biological activities, and biotechnological applications of Eclipta prostrata. Appl Microbiol Biotechnol. 2017;101(13):5247-57.

13. Sa F, Gao JL, Fung KP, Zheng Y, Lee SM, Wang YT. Anti-proliferative and pro-apoptotic effect of Smilax glabra Roxb. extract on hepatoma cell lines. Chem Biol Interact. 2008;171(1):1-14.

14. Cai Y, Tu J, Pan S, Jiang J, Shou Q, Ling Y, et al. Medicinal effect and its JP2/RyR2-based mechanism of Smilax glabra flavonoids on angiotensin II-induced hypertrophy model of cardiomyocytes. J Ethnopharmacol. 2015;169:435-40.

15. Wermuth CG. Multitargeted drugs: the end of the "one-target-one-disease" philosophy? Drug Discov Today. 2004;9(19):826-7.

16. Tao W, Xu X, Wang X, Li B, Wang Y, Li Y, et al. Network pharmacology-based prediction of the active ingredients and potential targets of Chinese herbal Radix Curcumae formula for application to cardiovascular disease. J Ethnopharmacol. 2013;145(1):1-10.

17. Huang C, Zheng C, Li Y, Wang Y, Lu A, Yang L. Systems pharmacology in drug discovery and therapeutic insight for herbal medicines. Brief Bioinform. 2014;15(5):710-33.

18. Lan T, Chang M, Li Q, Liang W, Yun C, Guo H. Optimization of Formation Process for Leigong Antihypertensive Granules by Central Composite Design-Respones Surface Methodology Based on CRITIC Weighted Evaluation. Journal of Chinese Medicinal Materials. 2019;42(6):1356-9.

19. Lan T, Li Q, Chang M, Yin C, Zhu D, Wu Z, et al. Lei-gong-gen formula granule attenuates hyperlipidemia in rats via cGMP-PKG signaling pathway. J Ethnopharmacol. 2020;260:112989.

20. Giudicelli JF, Freslon JL, Glasson S, Richer C. Captopril and hypertension development in the SHR. Clin Exp Hypertens. 1980;2(6):1083-96.

21. Ru J, Li P, Wang J, Zhou W, Li B, Huang C, et al. TCMSP: a database of systems pharmacology for drug discovery from herbal medicines. J Cheminform. 2014;6:13. 
22. Xue R, Fang Z, Zhang M, Yi Z, Wen C, Shi T. TCMID: Traditional Chinese Medicine integrative database for herb molecular mechanism analysis. Nucleic Acids Res. 2013;41(Database issue):D1089-95.

23. Liu Z, Guo F, Wang Y, Li C, Zhang X, Li H, et al. BATMAN-TCM: a Bioinformatics Analysis Tool for Molecular mechANism of Traditional Chinese Medicine. Sci Rep. 2016;6:21146.

24. Xu X, Zhang W, Huang C, Li Y, Yu H, Wang Y, et al. A novel chemometric method for the prediction of human oral bioavailability. Int J Mol Sci. 2012;13(6):6964-82.

25. Gong J, Cai C, Liu X, Ku X, Jiang H, Gao D, et al. ChemMapper: a versatile web server for exploring pharmacology and chemical structure association based on molecular 3D similarity method. Bioinformatics. 2013;29(14):1827-9.

26. Dai HJ, Wu JC, Tsai RT, Pan WH, Hsu WL. T-HOD: a literature-based candidate gene database for hypertension, obesity and diabetes. Database (Oxford). 2013;2013:bas061.

27. Shannon P, Markiel A, Ozier O, Baliga NS, Wang JT, Ramage D, et al. Cytoscape: a software environment for integrated models of biomolecular interaction networks. Genome Res. 2003;13(11):2498-504.

28. Szklarczyk D, Gable AL, Lyon D, Junge A, Wyder S, Huerta-Cepas J, et al. STRING v11: protein-protein association networks with increased coverage, supporting functional discovery in genome-wide experimental datasets. Nucleic Acids Res. 2019;47(D1):D607-D13.

29. Huang da W, Sherman BT, Lempicki RA. Systematic and integrative analysis of large gene lists using DAVID bioinformatics resources. Nat Protoc. 2009;4(1):44-57.

30. Livak KJ, Schmittgen TD. Analysis of relative gene expression data using real-time quantitative PCR and the 2(-Delta Delta C(T)) Method. Methods. 2001;25(4):402-8.

31. Arif M, Sadayappan S, Becker RC, Martin LJ, Urbina EM. Epigenetic modification: a regulatory mechanism in essential hypertension. Hypertens Res. 2019;42(8):1099-113.

32. Makani H, Bangalore S, Romero J, Htyte N, Berrios RS, Makwana H, et al. Peripheral edema associated with calcium channel blockers: incidence and withdrawal rate--a meta-analysis of randomized trials. J Hypertens. 2011;29(7):1270-80.

33. Raebel MA. Hyperkalemia associated with use of angiotensin-converting enzyme inhibitors and angiotensin receptor blockers. Cardiovasc Ther. 2012;30(3):e156-66.

34. Xiong X, Yang X, Liu Y, Zhang Y, Wang P, Wang J. Chinese herbal formulas for treating hypertension in traditional Chinese medicine: perspective of modern science. Hypertens Res. 2013;36(7):570-9.

35. Banquet S, Delannoy E, Agouni A, Dessy C, Lacomme S, Hubert F, et al. Role of G(i/o)-Src kinase$\mathrm{PI} 3 \mathrm{~K} /$ Akt pathway and caveolin-1 in beta(2)-adrenoceptor coupling to endothelial NO synthase in mouse pulmonary artery. Cell Signal. 2011;23(7):1136-43.

36. Shah DI, Singh M. Effect of demethylasterriquinone b1 in hypertension associated vascular endothelial dysfunction. Int J Cardiol. 2007;120(3):317-24. 
37. Yang Q, Xue HM, Wong WT, Tian XY, Huang Y, Tsui SK, et al. AVE3085, an enhancer of endothelial nitric oxide synthase, restores endothelial function and reduces blood pressure in spontaneously hypertensive rats. Br J Pharmacol. 2011;163(5):1078-85.

38. Frame MC. Src in cancer: deregulation and consequences for cell behaviour. Biochim Biophys Acta. 2002;1602(2):114-30.

39. Paravicini TM, Montezano AC, Yusuf H, Touyz RM. Activation of vascular p38MAPK by mechanical stretch is independent of c-Src and NADPH oxidase: influence of hypertension and angiotensin II. J Am Soc Hypertens. 2012;6(3):169-78.

40. He H, Venema VJ, Gu X, Venema RC, Marrero MB, Caldwell RB. Vascular endothelial growth factor signals endothelial cell production of nitric oxide and prostacyclin through flk-1/KDR activation of cSrc. J Biol Chem. 1999;274(35):25130-5.

41. Xing W, Yan W, Liu P, Ji L, Li Y, Sun L, et al. A novel mechanism for vascular insulin resistance in normotensive young SHRs: hypoadiponectinemia and resultant APPL1 downregulation. Hypertension. 2013;61(5):1028-35.

42. Yamamoto E, Yamashita T, Tanaka T, Kataoka K, Tokutomi Y, Lai ZF, et al. Pravastatin enhances beneficial effects of olmesartan on vascular injury of salt-sensitive hypertensive rats, via pleiotropic effects. Arterioscler Thromb Vasc Biol. 2007;27(3):556-63.

43. Nemoto S, Kobayashi T, Taguchi K, Matsumoto T, Kamata K. Losartan improves aortic endotheliumdependent relaxation via proline-rich tyrosine kinase 2/Src/Akt pathway in type 2 diabetic GotoKakizaki rats. Am J Physiol Heart Circ Physiol. 2011;301(6):H2383-94.

44. Chong KP, Rossall S, Atong M. In Vitro Antimicrobial Activity and Fungitoxicity of Syringic Acid, Caffeic Acid and 4-hydroxybenzoic Acid against Ganoderma Boninense. Journal of Agricultural Science. 2009;1(2).

45. Figueiredo-Gonzalez M, Reboredo-Rodriguez P, Gonzalez-Barreiro C, Simal-Gandara J, Valentao P, Carrasco-Pancorbo A, et al. Evaluation of the neuroprotective and antidiabetic potential of phenolrich extracts from virgin olive oils by in vitro assays. Food Res Int. 2018;106:558-67.

46. Ling WC, Ahmad F, Mat Ali R. Luteolin and 4-Hydroxybenzoic Acid from the Leaves Vitex negundo. 2005.

47. Chaudhary J, Jain A, Manuja R, Sachdeva S. A Comprehensive Review on Biological activities of phydroxy benzoic acid and its derivatives. International Journal of Pharmaceutical Sciences Review \& Research. 2013;22(2).

48. Leeya Y, Mulvany MJ, Queiroz EF, Marston A, Hostettmann K, Jansakul C. Hypotensive activity of an n-butanol extract and their purified compounds from leaves of Phyllanthus acidus (L.) Skeels in rats. Eur J Pharmacol. 2010;649(1-3):301-13.

49. Srinivasulu C, Ramgopal M, Ramanjaneyulu G, Anuradha CM, Suresh Kumar C. Syringic acid (SA) A Review of Its Occurrence, Biosynthesis, Pharmacological and Industrial Importance. Biomed Pharmacother. 2018;108:547-57. 
50. Jalili T, Carlstrom J, Kim S, Freeman D, Jin H, Wu TC, et al. Quercetin-supplemented diets lower blood pressure and attenuate cardiac hypertrophy in rats with aortic constriction. J Cardiovasc Pharmacol. 2006;47(4):531-41.

51. Kiran p, Denn.i M, Daniel M. Antidiabetic Principles, Phospholipids And Fixed Oil of Kodo Millet (Paspalum scrobiculatum Linn.). Indian Journal of Applied Research. 2011;4:13-5.

52. Kumar S, Prahalathan P, Raja B. Syringic acid ameliorates (L)-NAME-induced hypertension by reducing oxidative stress. Naunyn Schmiedebergs Arch Pharmacol. 2012;385(12):1175-84.

53. Benmalek Y, Yahia OA, Belkebir A, Fardeau ML. Anti-microbial and anti-oxidant activities of Illicium verum, Crataegus oxyacantha ssp monogyna and Allium cepa red and white varieties. Bioengineered. 2013;4(4):244-8.

54. Nakamura T, Okuyama E, Yamazaki M. Neurotropic components from star anise (Illicium verum Hook. fil.). Chem Pharm Bull (Tokyo). 1996;44(10):1908-14.

55. Thring TS, Hili P, Naughton DP. Anti-collagenase, anti-elastase and anti-oxidant activities of extracts from 21 plants. BMC Complement Altern Med. 2009;9:27.

56. Rabelo TK, Guimaraes AG, Oliveira MA, Gasparotto J, Serafini MR, de Souza Araujo AA, et al. Shikimic acid inhibits LPS-induced cellular pro-inflammatory cytokines and attenuates mechanical hyperalgesia in mice. Int Immunopharmacol. 2016;39:97-105.

57. Arif T. Salicylic acid as a peeling agent: a comprehensive review. Clin Cosmet Investig Dermatol. 2015;8:455-61.

58. Han J, He GS, Liu JW, Peter A, Chen PP. Anti-oxidant Effects of Xinjiang Red Raspberry Fruit Extracts in Spontaneously Hypertensive Rats. Chinese Journal of Hypertension. 2009;17(8):695-9.

59. Meyers CD, Kamanna VS, Kashyap ML. Niacin therapy in atherosclerosis. Curr Opin Lipidol. 2004;15(6):659-65.

60. Cho KH, Kim HJ, Rodriguez-Iturbe B, Vaziri ND. Niacin ameliorates oxidative stress, inflammation, proteinuria, and hypertension in rats with chronic renal failure. Am J Physiol Renal Physiol. 2009;297(1):F106-13.

61. Cho KH, Kim HJ, Kamanna VS, Vaziri ND. Niacin improves renal lipid metabolism and slows progression in chronic kidney disease. Biochim Biophys Acta. 2010;1800(1):6-15.

62. Linck VM, da Silva AL, Figueiro M, Piato AL, Herrmann AP, Dupont Birck F, et al. Inhaled linaloolinduced sedation in mice. Phytomedicine. 2009;16(4):303-7.

63. Pereira I, Severino P, Santos AC, Silva AM, Souto EB. Linalool bioactive properties and potential applicability in drug delivery systems. Colloids Surf B Biointerfaces. 2018;171:566-78.

64. Camargo SB, Simoes LO, Medeiros CFA, de Melo Jesus A, Fregoneze JB, Evangelista A, et al. Antihypertensive potential of linalool and linalool complexed with beta-cyclodextrin: Effects of subchronic treatment on blood pressure and vascular reactivity. Biochem Pharmacol. 2018;151:3846. 
65. Vera-Aviles M, Vantana E, Kardinasari E, Koh NL, Latunde-Dada GO. Protective Role of Histidine Supplementation Against Oxidative Stress Damage in the Management of Anemia of Chronic Kidney Disease. Pharmaceuticals (Basel). 2018;11(4).

66. Tuttle KR, Milton JE, Packard DP, Shuler LA, Short RA. Dietary amino acids and blood pressure: a cohort study of patients with cardiovascular disease. Am J Kidney Dis. 2012;59(6):803-9.

67. Toba H, Nakamori A, Tanaka Y, Yukiya R, Tatsuoka K, Narutaki M, et al. Oral L-histidine exerts antihypertensive effects via central histamine $\mathrm{H} 3$ receptors and decreases nitric oxide content in the rostral ventrolateral medulla in spontaneously hypertensive rats. Clin Exp Pharmacol Physiol. 2010;37(1):62-8.

\section{Tables}

Table 1: Primer sequence used for PCR analysis

\begin{tabular}{lll}
\hline \multicolumn{1}{c}{ Gene } & \multicolumn{1}{c}{ Forward } & \multicolumn{1}{c}{ Reverse } \\
\hline NOS3 & 5'-CAGAGATTGGCATGAGGGACC-3' & 5'-TCCACAGTGATGAGGTTGTCC-3' \\
SRC & 5'-CAAGATCACTAGACGGGAATCAG-3' & 5'-GTTTCACATTTAGGCCCTTGG-3' \\
PTGS2 & 5'-ATCAGAACCGCATTGCCTCT-3' & 5'-GCCAGCAATCTGTCTGGTGA-3' \\
NGF & 5'-GTCTGGGCCCAATAAAGGCT-3' & 5'-TGTACGCCGATCAAAAACGC-3' \\
VEGFA & 5'-CGTCCTGTGTGCCCCTAATG-3' & 5'-TGTGCTGGCTTTGGTGAGGT-3' \\
EGF & 5'-CTACTACAGGACTCGGAAGCAG-3' & 5'-GTTGGGGACCAGAAGACACC-3' \\
EGFR & 5'-AGCCGTCCTGTCCAACTATG-3' & 5'-TTGCTAAATCGCACAGCACC-3' \\
ERK2 & 5'-ATTGGTCAGGACAAGGGCTCA-3' & 5'-CCACTACGACCAGAACTGCC-3' \\
CCL2 & 5'-TGCAGGTCTCTGTCACGCTTC-3' & 5'-TTCTCCAGCCGACTCATTGG-3' \\
NPY & 5'-GCCAGATACTACTCCGCTCTG-3' & 5'-GTCTCAGGGCTGGATCTCTTG-3' \\
ESR1 & 5'-CAGACAGGGAGCTGGTTCATA-3' & 5'-GCACACTCCAGAAGGTGAACT-3' \\
TH & 5'-GTCTCAGAGCAGGATGCCAAG-3' & 5'-ATCCTCGATGAGACTCTGTCGC-3' \\
B-actin & 5'-GTCAGGTCATCACTATCGGCAAT-3' & 5'-AGAGGTCTTTACGGATGTCAACGT-3' \\
\hline
\end{tabular}

Table 2: The information of 12 key targets between compounds and hypertension targets 


\begin{tabular}{|c|c|c|c|}
\hline Gene & Compounds & Gene & Compounds \\
\hline VEGFA & glucoside & MAPK1 & Cedar acid \\
\hline NPY & Ferulic Acid & MAPK1 & Ferulic Acid \\
\hline EGF & salicylic acid & NOS3 & L-Bornyl acetate \\
\hline EGF & nicotinic acid & NOS3 & p-Hydroxybenzoic acid \\
\hline EGF & p-Hydroxybenzoic acid & NOS3 & salicylic acid \\
\hline EGFR & naringenin & NOS3 & Pulegone \\
\hline $\mathrm{TH}$ & $(1 \mathrm{R}, 2 \mathrm{R}, 5 \mathrm{~S})-2,6,6$-trimethylnorpinan-3-one & NOS3 & Cedar acid \\
\hline $\mathrm{TH}$ & Ferulic Acid & NOS3 & nicotinic acid \\
\hline $\mathrm{TH}$ & Histidine & NOS3 & Histidine \\
\hline NGF & (-)-alpha-Pinene & NOS3 & (R)-linalool \\
\hline NGF & p-Hydroxybenzoic acid & NOS3 & 5-Hydroxymethylfurfural \\
\hline NGF & (-)-nopinene & ESR1 & L-Bornyl acetate \\
\hline SRC & Shikimic Acid & ESR1 & $\begin{array}{l}(1 \mathrm{R}, 2 \mathrm{R}, 5 \mathrm{~S})-2,6,6 \text {-trimethylnorpinan- } \\
\text { 3-one }\end{array}$ \\
\hline SRC & nicotinic acid & ESR1 & Pratensein \\
\hline SRC & Histidine & ESR1 & alpha-Terthienyl methyl acetate \\
\hline PTGS2 & naringenin & ESR1 & diosgenin \\
\hline PTGS2 & Histidine & ESR1 & (+)-Isoborneol \\
\hline PTGS2 & demethylwedelolactone & ESR1 & sitosterol \\
\hline PTGS2 & acacetin & ESR1 & Stigmasterol \\
\hline PTGS2 & Ferulic Acid & ESR1 & butin \\
\hline PTGS2 & Xanthanoic acid & ESR1 & Baimuxinol \\
\hline PTGS2 & 5-Hydroxymethylfurfural & ESR1 & $\begin{array}{l}\text { (Z)-1-(2,4-dihydroxyphenyl)-3- } \\
\text { (3,4-dihydroxyphenyl)prop-2-en-1-one }\end{array}$ \\
\hline PTGS2 & (L)-alpha-Terpineol & ESR1 & (-)-nopinene \\
\hline PTGS2 & $(1 \mathrm{R}, 2 \mathrm{R}, 5 \mathrm{~S})-2,6,6$-trimethylnorpinan-3-one & ESR1 & 1,8-cineole \\
\hline PTGS2 & Pulegone & ESR1 & (-)-alpha-Pinene \\
\hline PTGS2 & MENTHOL & ESR1 & demethylwedelolactone \\
\hline PTGS2 & L-Limonen & ESR1 & 3'-O-Methylorobol \\
\hline PTGS2 & salicylic acid & ESR1 & wedelolactone \\
\hline PTGS2 & nicotinic acid & ESR1 & luteolin \\
\hline PTGS2 & p-Hydroxybenzoic acid & ESR1 & acacetin \\
\hline PTGS2 & L-Bornyl acetate & ESR1 & Dihydroresveratrol \\
\hline PTGS2 & Cedar acid & ESR1 & naringenin \\
\hline CCL2 & Ferulic Acid & ESR1 & cis-resveratrol \\
\hline MAPK1 & p-Hydroxybenzoic acid & & \\
\hline
\end{tabular}

Table 3: Identify of the compounds of LFG by UPLC- Q-TOF/MS analysis in negative ion mode 


\begin{tabular}{|c|c|c|c|c|c|c|c|}
\hline NO. & R-T & $\mathrm{m} / \mathrm{z}$ & MS/MS & Elemental composition & Ion form & ppm & Compounds \\
\hline 1 & 3.73 & 173.0458 & 93.0354 & $\mathrm{C}_{7} \mathrm{H}_{10} \mathrm{O}_{5}$ & {$[\mathrm{M}-\mathrm{H}]^{-}$} & -1.3 & Shikimic acid \\
\hline 2 & 3.90 & 124.0394 & $78.0334 / 80.0490$ & $\mathrm{C}_{6} \mathrm{H}_{5} \mathrm{NO}_{2}$ & {$[\mathrm{M}-\mathrm{H}]^{+}$} & -0.7 & Nicotinic acid \\
\hline 3 & 18.59 & 137.0244 & 93.0354 & $\mathrm{C}_{7} \mathrm{H}_{6} \mathrm{O}_{3}$ & {$[\mathrm{M}-\mathrm{H}]^{-}$} & 0.2 & p-Hydroxybenzoic acid \\
\hline 4 & 19.72 & 353.0882 & 191.0565 & $\mathrm{C}_{16} \mathrm{H}_{18} \mathrm{O}_{9}$ & {$[\mathrm{M}-\mathrm{H}]^{-}$} & -1.0 & Chlorogenic acid \\
\hline 5 & 21.78 & 353.0881 & $191.0563 / 173.0456$ & $\mathrm{C}_{16} \mathrm{H}_{18} \mathrm{O}_{9}$ & {$[\mathrm{M}-\mathrm{H}]^{-}$} & -0.9 & 4-Dicaffeoylquinic acid \\
\hline 6 & 28.69 & 515.1196 & $191.0563 / 179.0351$ & $\mathrm{C}_{25} \mathrm{H}_{24} \mathrm{O}_{12}$ & {$[\mathrm{M}-\mathrm{H}]^{-}$} & -0.1 & Isochlorogenic acid C \\
\hline 7 & 35.37 & 193.0509 & $106.0438 / 134.0376$ & $\mathrm{C}_{10} \mathrm{H}_{10} \mathrm{O}_{4}$ & {$[\mathrm{M}-\mathrm{H}]^{-}$} & -1.4 & Ferulic acid \\
\hline 8 & 36.84 & 449.1089 & $151.0036 / 285.0405$ & $\mathrm{C}_{21} \mathrm{H}_{22} \mathrm{O}_{11}$ & {$[\mathrm{M}-\mathrm{H}]^{-}$} & 0.1 & Neoastilbin \\
\hline 9 & 38.09 & 449.1099 & $151.0040 / 285.0413$ & $\mathrm{C}_{21} \mathrm{H}_{22} \mathrm{O}_{11}$ & {$[\mathrm{M}-\mathrm{H}]^{-}$} & -2.3 & Astilbin \\
\hline 10 & 40.67 & 449.1095 & $151.0037 / 285.0410$ & $\mathrm{C}_{21} \mathrm{H}_{22} \mathrm{O}_{11}$ & {$[\mathrm{M}-\mathrm{H}]^{-}$} & -1.3 & Neoisoastilbin \\
\hline 11 & 41.50 & 449.1094 & $151.0037 / 285.0408$ & $\mathrm{C}_{21} \mathrm{H}_{22} \mathrm{O}_{11}$ & {$[\mathrm{M}-\mathrm{H}]^{-}$} & -0.9 & Isoastilbin \\
\hline 12 & 43.45 & 433.1147 & $180.0068 / 269.0463$ & $\mathrm{C}_{21} \mathrm{H}_{22} \mathrm{O}_{10}$ & {$[\mathrm{M}-\mathrm{H}]^{-}$} & -1.6 & Engeletin \\
\hline 13 & 47.33 & 433.1137 & $180.0059 / 269.0448$ & $\mathrm{C}_{21} \mathrm{H}_{22} \mathrm{O}_{10}$ & {$[\mathrm{M}-\mathrm{H}]^{-}$} & -0.4 & Isoengelitin \\
\hline 14 & 49.31 & 137.0245 & 93.0354 & $\mathrm{C}_{7} \mathrm{H}_{6} \mathrm{O}_{3}$ & {$[\mathrm{M}-\mathrm{H}]^{-}$} & -0.5 & Salicylic acid \\
\hline 15 & 55.45 & 285.0409 & $133.0297 / 151.0033$ & $\mathrm{C}_{15} \mathrm{H}_{10} \mathrm{O}_{6}$ & {$[\mathrm{M}-\mathrm{H}]^{-}$} & -1.7 & Luteolin \\
\hline 16 & 56.15 & 313.0368 & 298.0126 & $\mathrm{C}_{16} \mathrm{H}_{10} \mathrm{O}_{7}$ & {$[\mathrm{M}-\mathrm{H}]^{-}$} & -4.4 & Wedelolactone \\
\hline 17 & 59.00 & 503.3381 & $389.2861 / 437.3066$ & $\mathrm{C}_{30} \mathrm{H}_{48} \mathrm{O}_{6}$ & {$[\mathrm{M}-\mathrm{H}]^{-}$} & -0.6 & Madecassic-acid \\
\hline 18 & 60.20 & 487.3431 & $379.3034 / 409.3156$ & $\mathrm{C}_{30} \mathrm{H}_{48} \mathrm{O}_{5}$ & {$[\mathrm{M}-\mathrm{H}]^{-}$} & -0.4 & Asiatic acid \\
\hline
\end{tabular}

\section{Figures}

\section{Step1: Animal experiment}

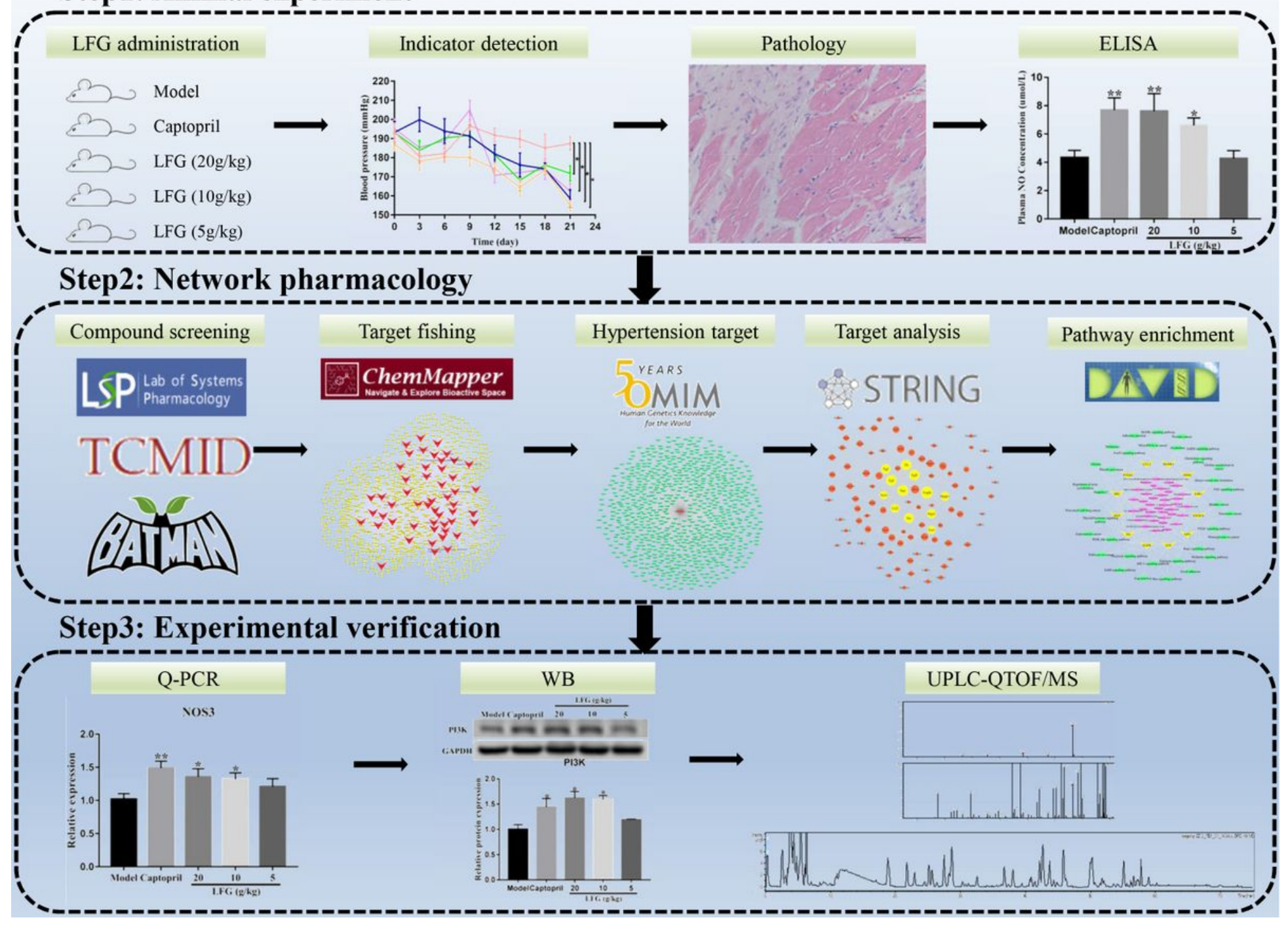


Figure 1

Work flow of the study.
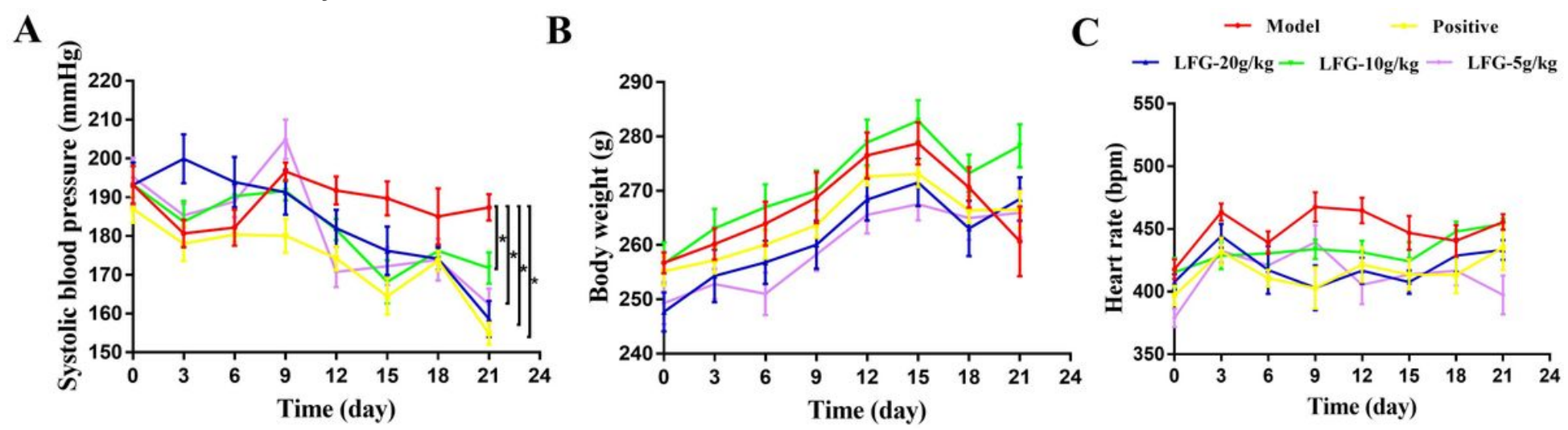

\section{Figure 2}

Effect of LFG on blood pressure, body weight and heart rate of SHR. (A) Blood pressure changes; (B) body weight changes; (C) heart rate changes. Compared with model group, ${ }^{*} \mathrm{P}<0.05$.

A

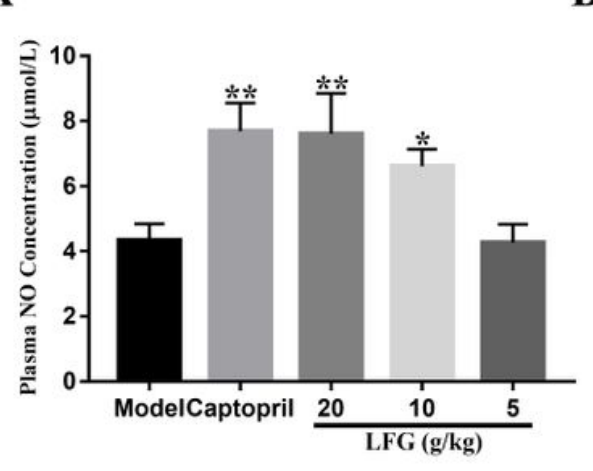

B

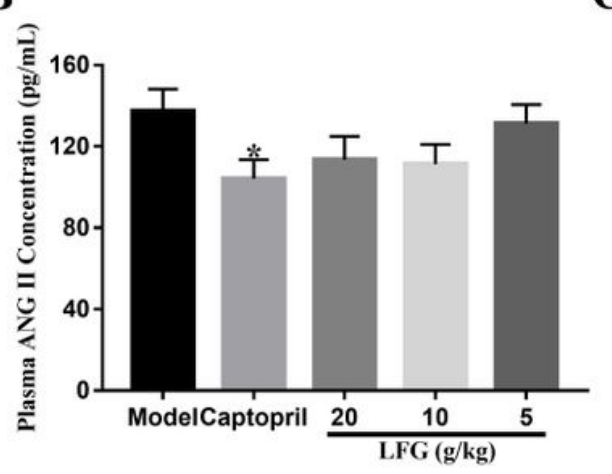

C

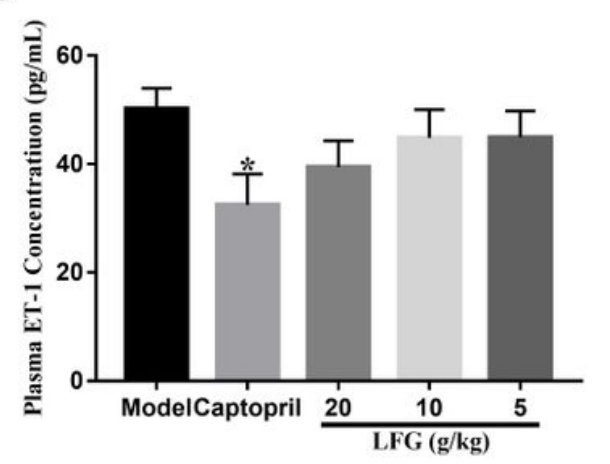

Figure 3

Effect of LFG on (A) NO, (B) Ang $\otimes$ and (C) ET-1 levels in serum of SHR. Compared with model group, * $P$ $<0.05, * * \mathrm{P}<0.01$. 
A

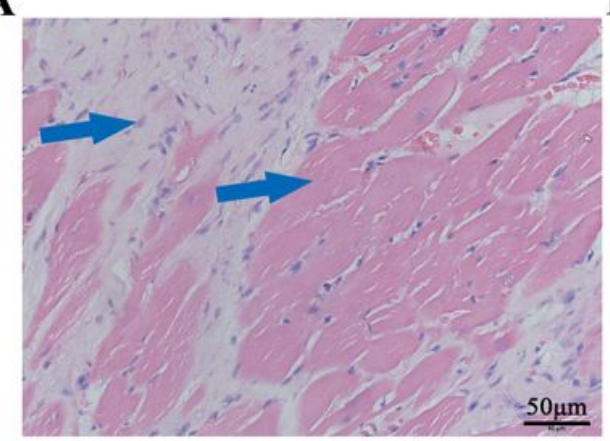

D

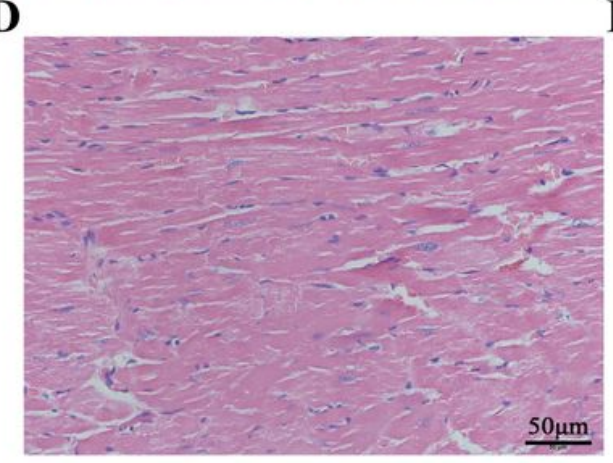

B

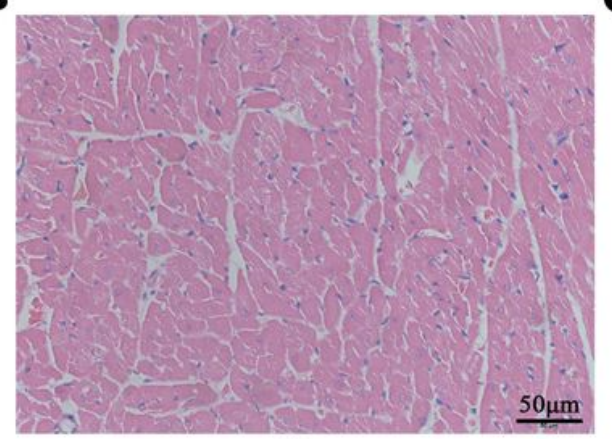

$\mathbf{E}$

C

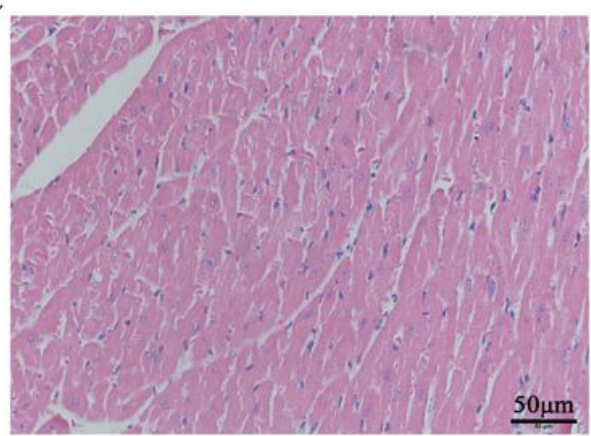

$50 \mu \mathrm{m}$

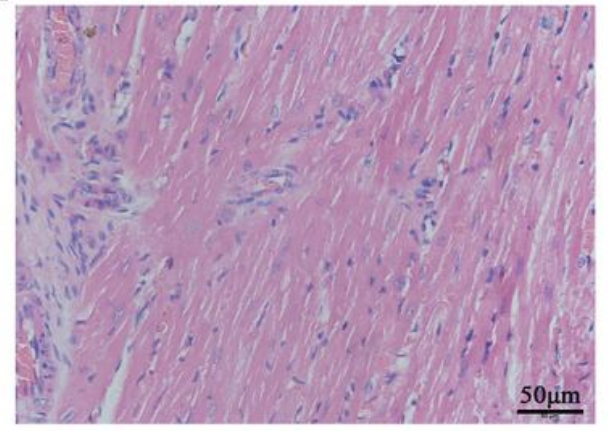

\section{Figure 4}

H\&E staining results of heart tissue. (A) Model group; (B) Captopril group; (C) High-dose group; (D) Middle-dose group; (E) Low-dose group. Magnification 400x, scale bar $=50 \mu \mathrm{m}$. The blue arrows indicate cardiac hypertrophy and fibrosis. 

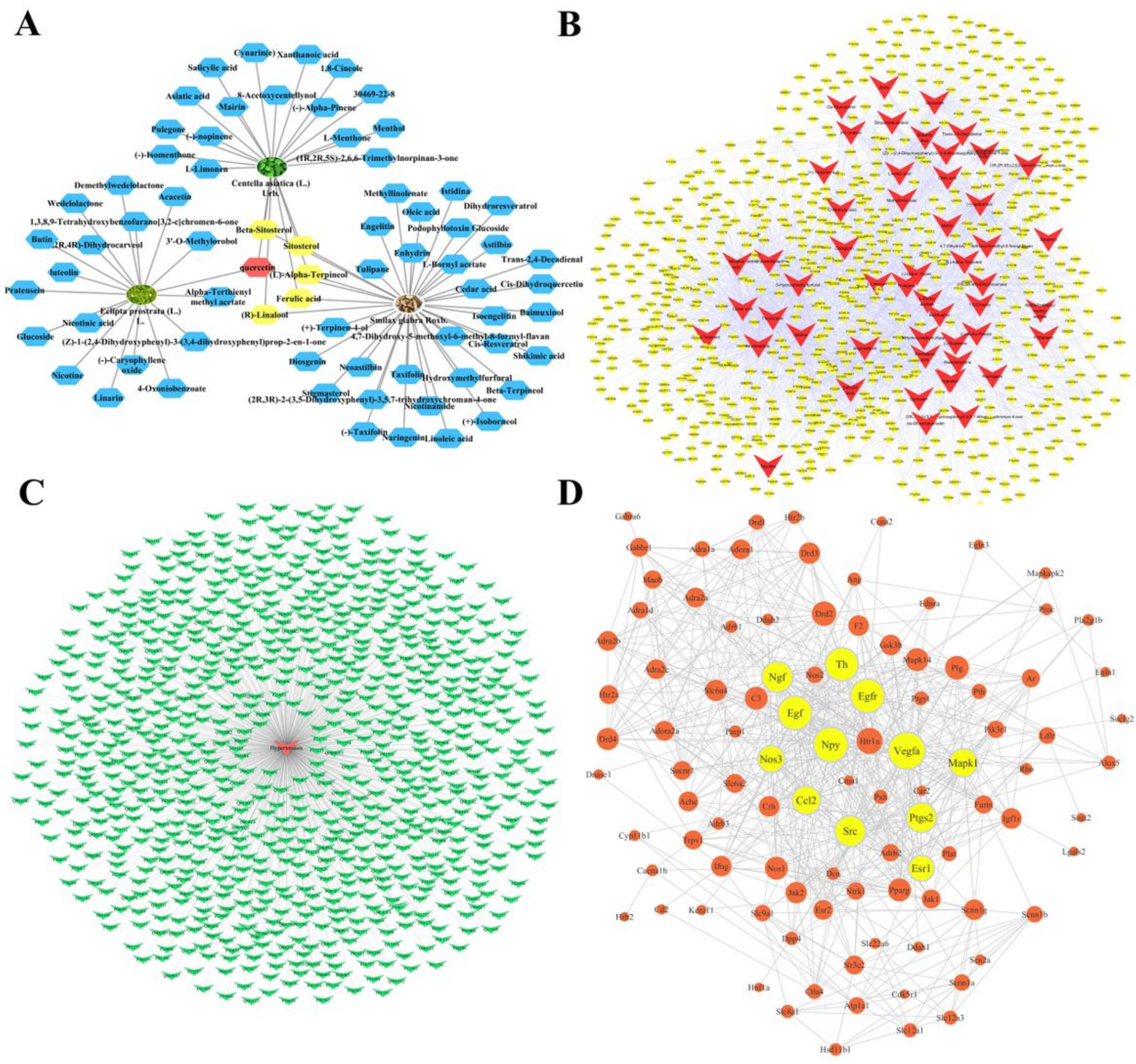

\section{Figure 5}

The results of network pharmacology prediction. (A) The herb-compound network. The ellipse nodes represent herbs, and the hexagon nodes are compounds. Among these compounds, yellow parts are the common components of Centella asiatica (L.) Urb. and Smilax glabra Roxb., and quercetin (red hexagon) is common compounds of 3 herbs. (B) The compound-target network. The $V$ shape nodes represent 53 compounds, and the 765 yellow nodes are targets corresponding to the compounds. (C) The hypertension-target network. In this figure, the red node in the center is hypertension, and the green nodes represent gene targets. (D) The protein-protein interaction network within the common targets. It shows 
the relationship between 101 common targets. The size of nodes changes from big to small according to degree values, and the 12 yellow nodes are key targets after screening.

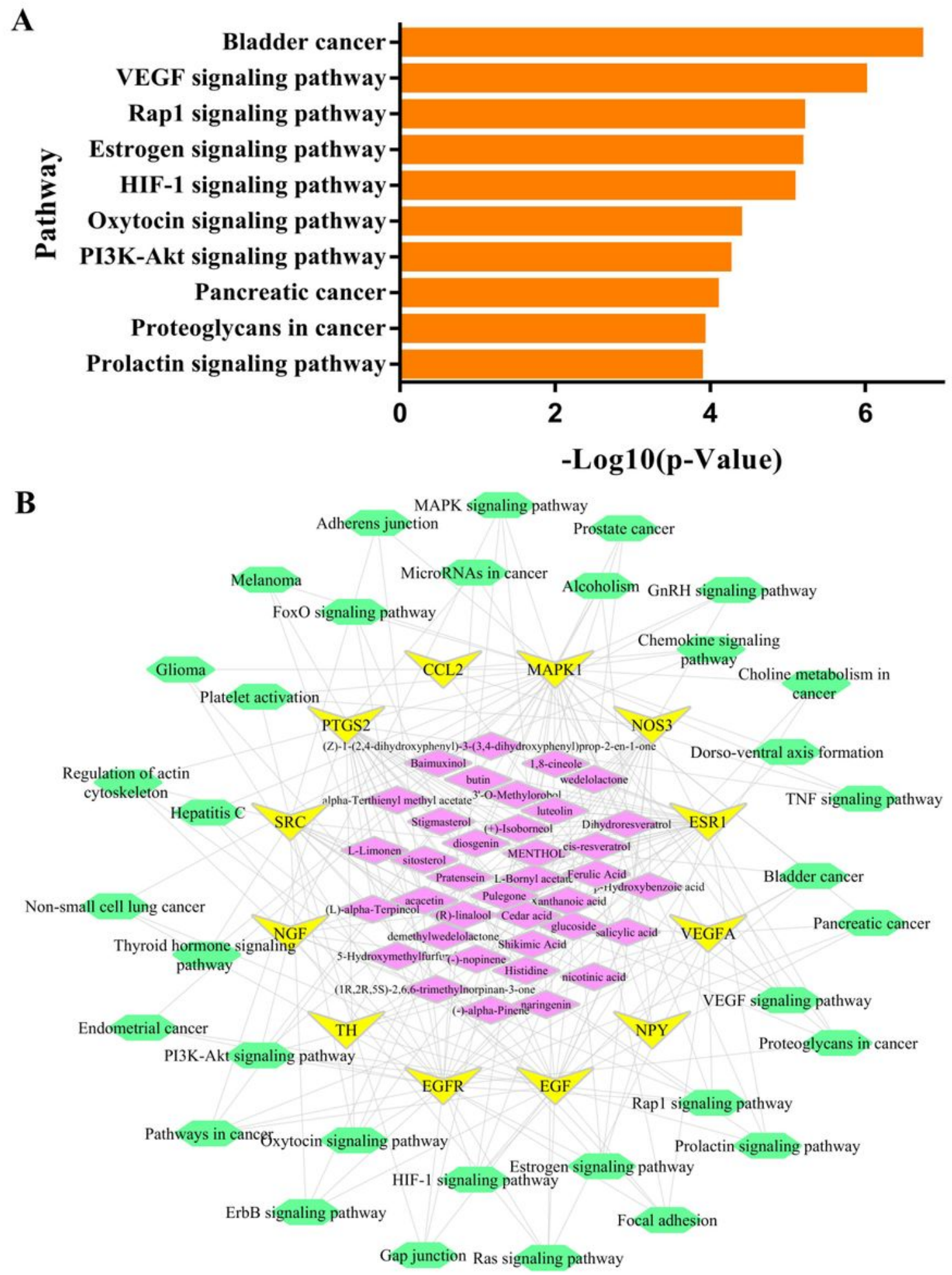

\section{Figure 6}

(A) The relationship between TOP10 pathways and their -log10 (P). (B) The compound-target-pathway network. Compounds are indicated by 37 purple nodes; gene targets are shown as 12 yellow nodes; pathways are labeled with 34 green nodes. 
A

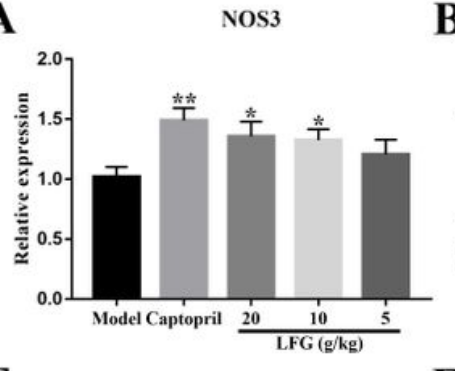

$\mathbf{E}$

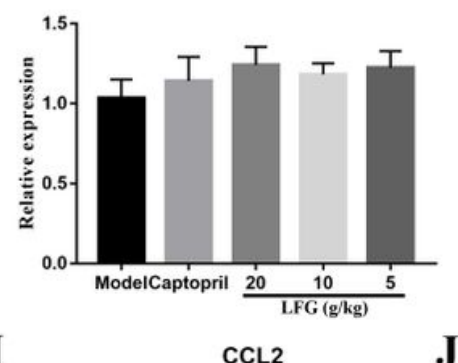

I

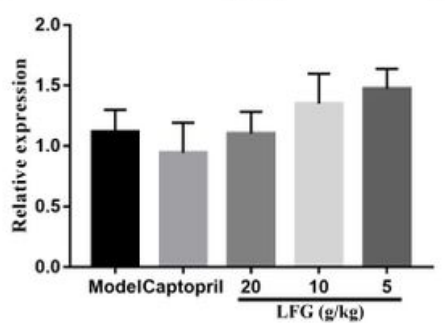

B

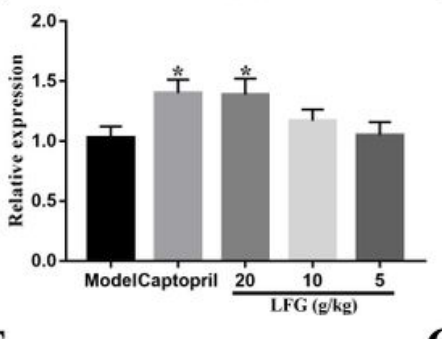

C

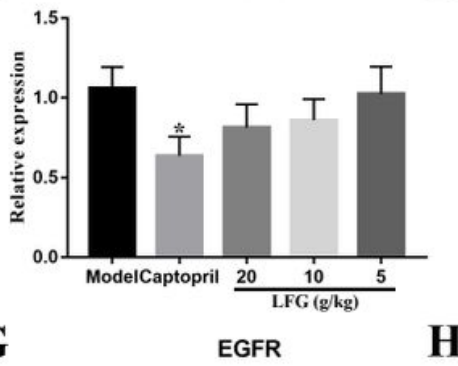

D NGF
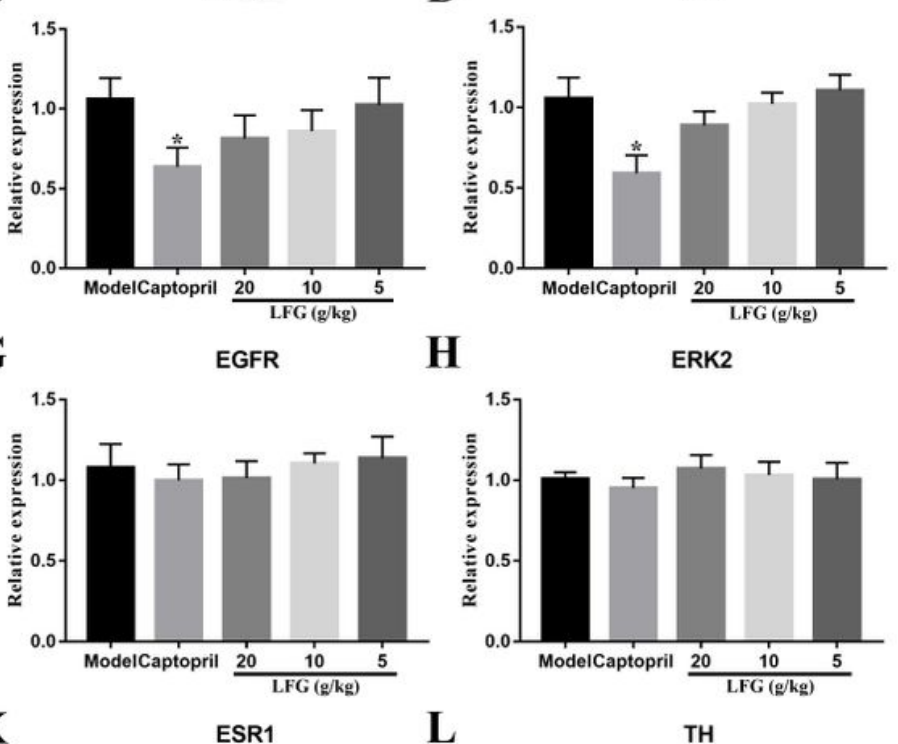

$\mathbf{L}$

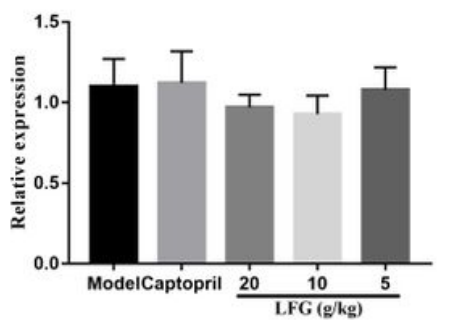

TH

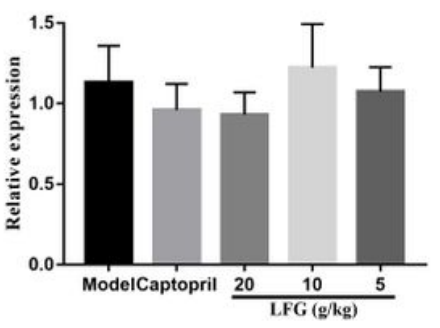

Figure 7

Effect of LFG on mRNA expression levels of key targets. (A) NOS3; (B) SRC; (C) PTGS2; (D) NGF; (E) VEGFA; (F) EGF; (G) EGFR; (H) ERK2; (I) CCL2; (J) NPY; (K) ESR1; (L) TH. Compared with model group, $\star P<0.05 \rrbracket * * P<0.01$. 
A

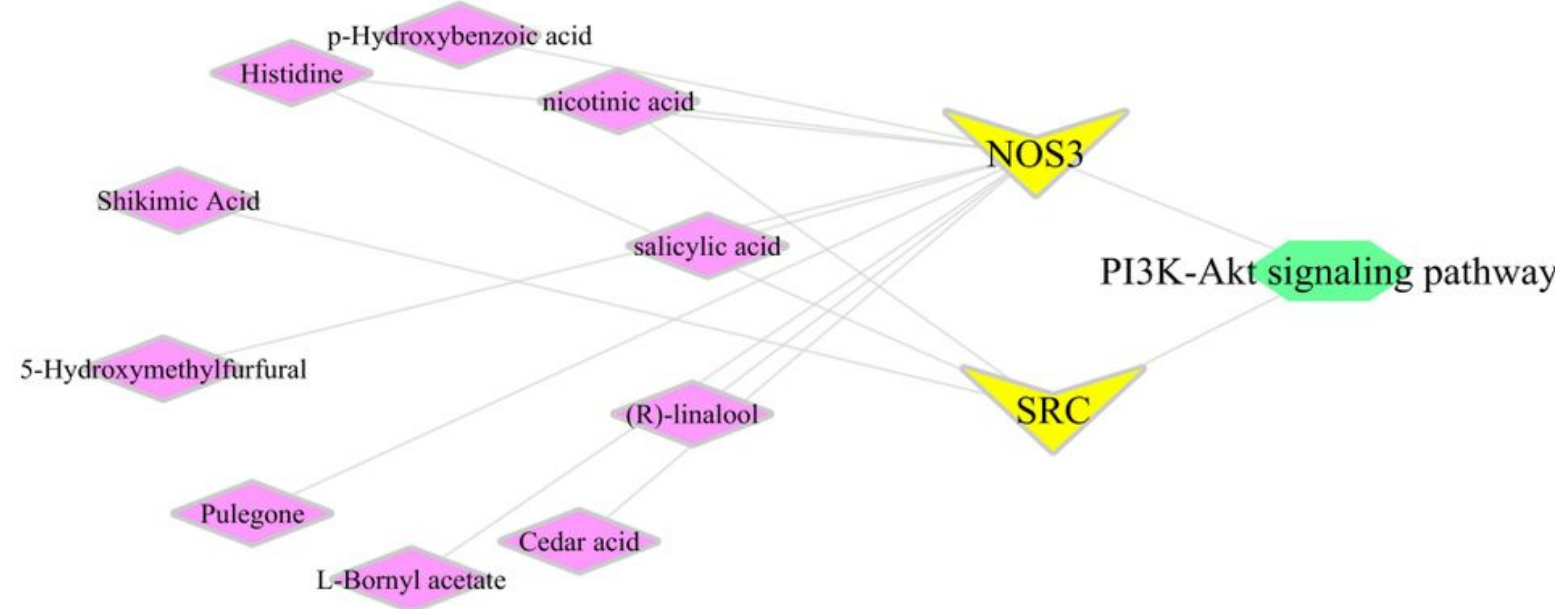

B

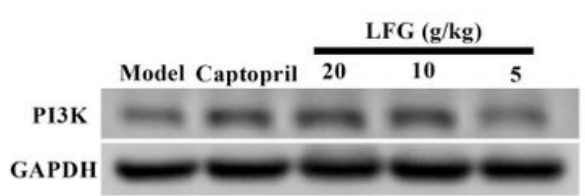

PI3K

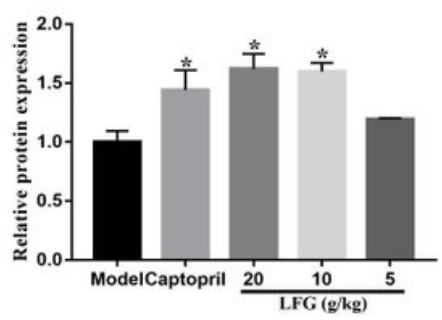

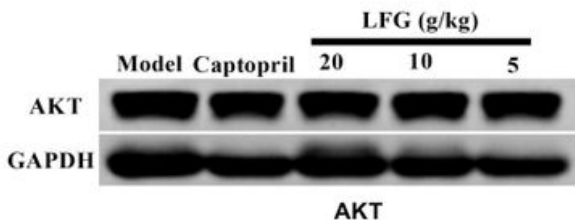

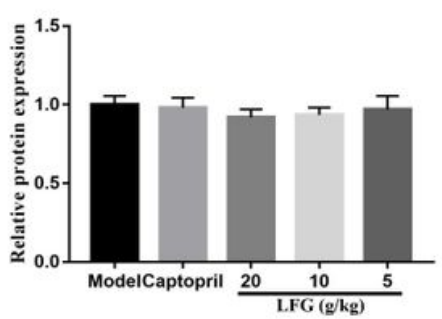

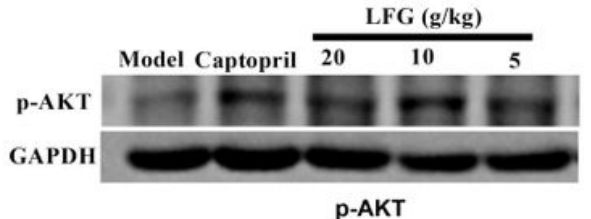

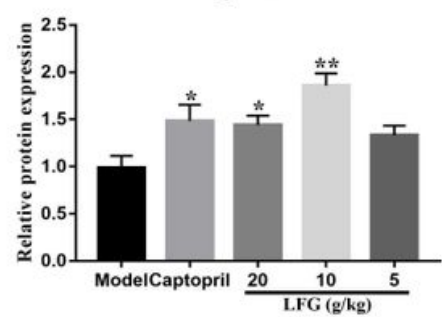

\section{Figure 8}

(A) The compound-target-pathway network of significant genes ( $V$ nodes). Compounds are indicated by 10 diamond nodes; pathways are shown as hexagon nodes. (B) PI3K, AKT and p-AKT protein expression levels among different groups. Compared with model group, ${ }^{\star} P<0.05$, $* \star P<0.01$. 
$\mathbf{A}$

E

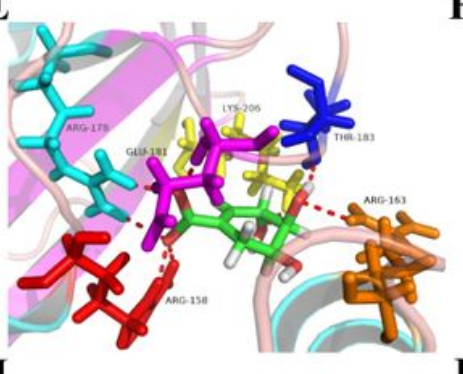

I

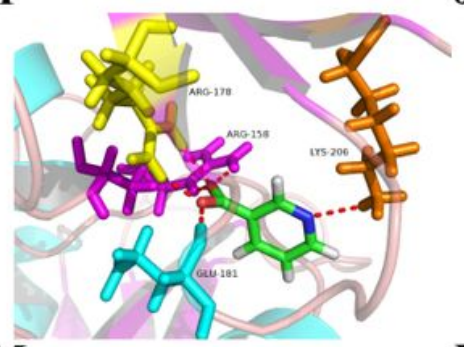

M

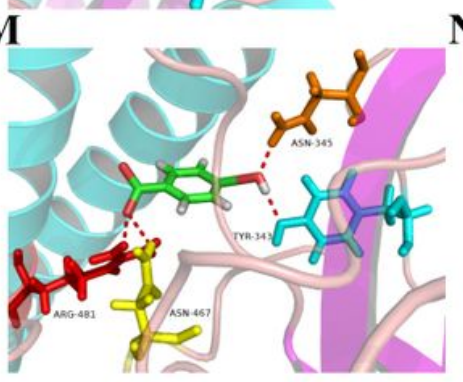

B
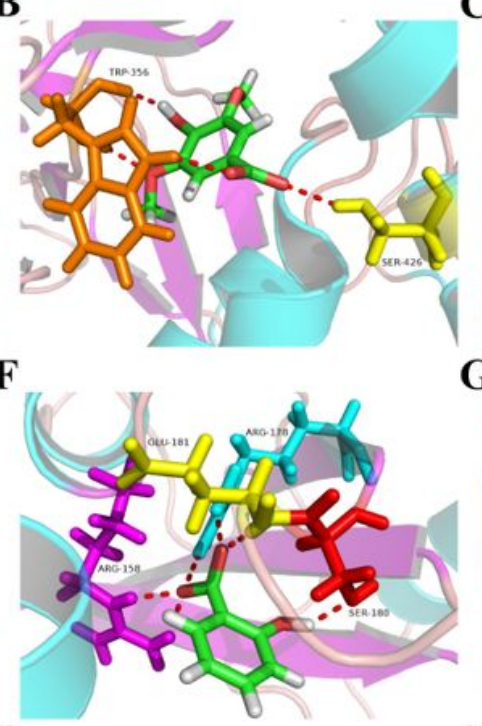

J

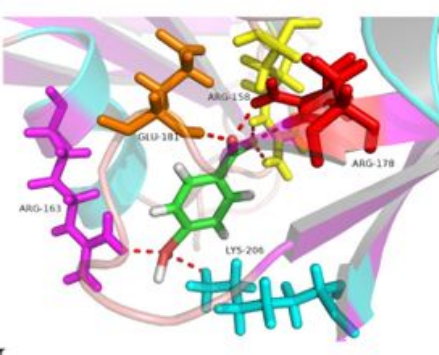

C

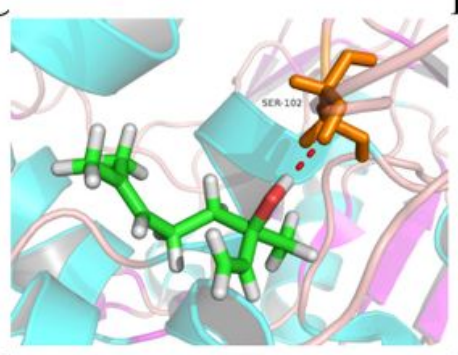

G

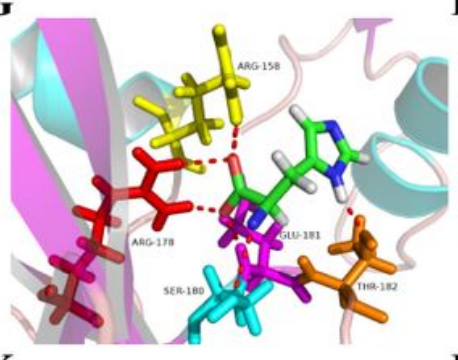

K

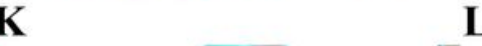

D

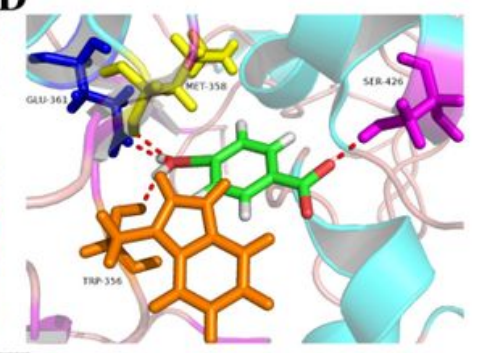

H

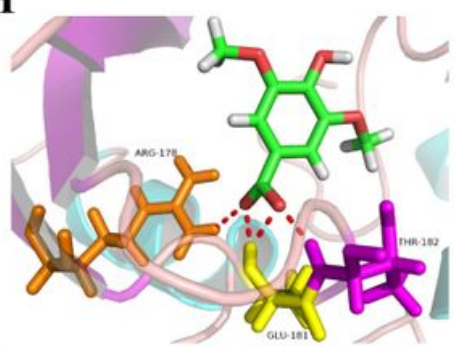

L

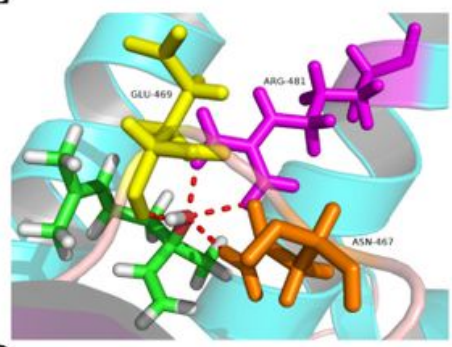

N

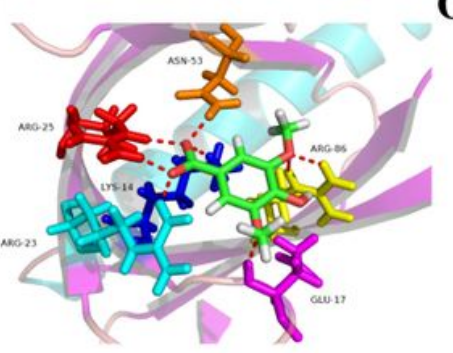

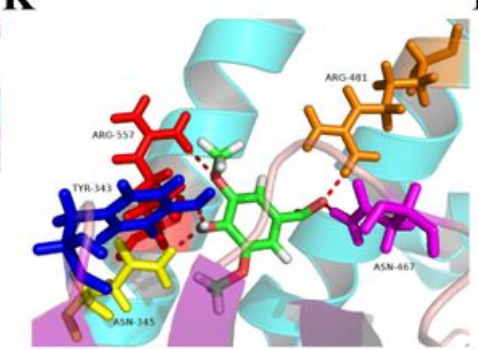

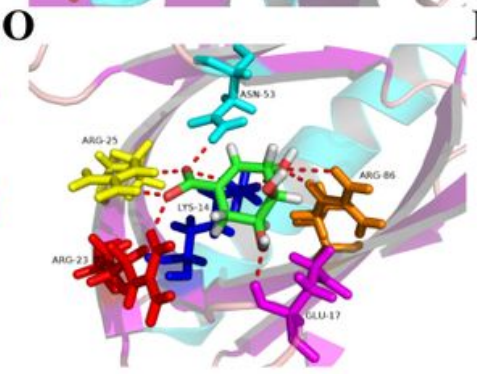

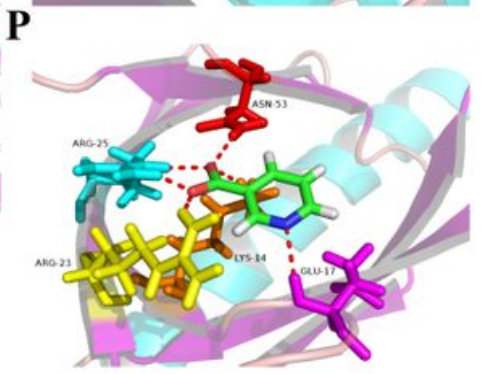

Figure 9

The binding modes of compounds and NOS3: (A) Histidine, (B) Cedar acid, (C) Linalool, (D) pHydroxybenzoic acid. The binding modes of compounds and SRC: (E) Shikimic acid, (F) Salicylic acid, (G) Histidine, $(\mathrm{H})$ Cedar acid, (I) Nicotinic acid, (J) p-Hydroxybenzoic acid. The binding modes of compounds and PI3K: (K) Cedar acid, (L) p-Hydroxybenzoic acid, (M) Linalool. The binding modes of compounds and AKT: (N) Cedar acid, (O) Shikimic acid, (P) Nicotinic acid. 


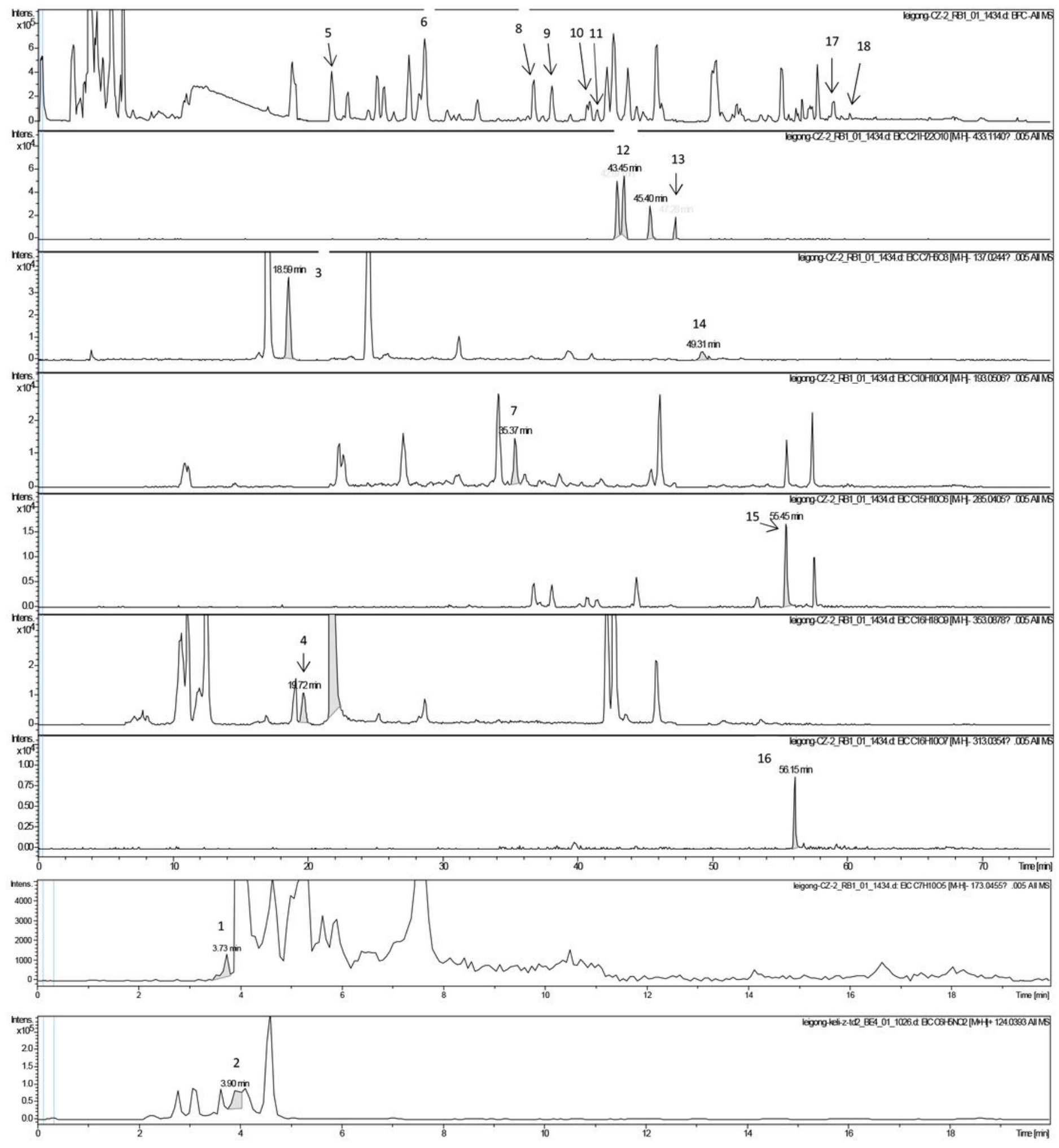

Figure 10

UPLC-QTOF/MS BPI chromatograms of LFG. 


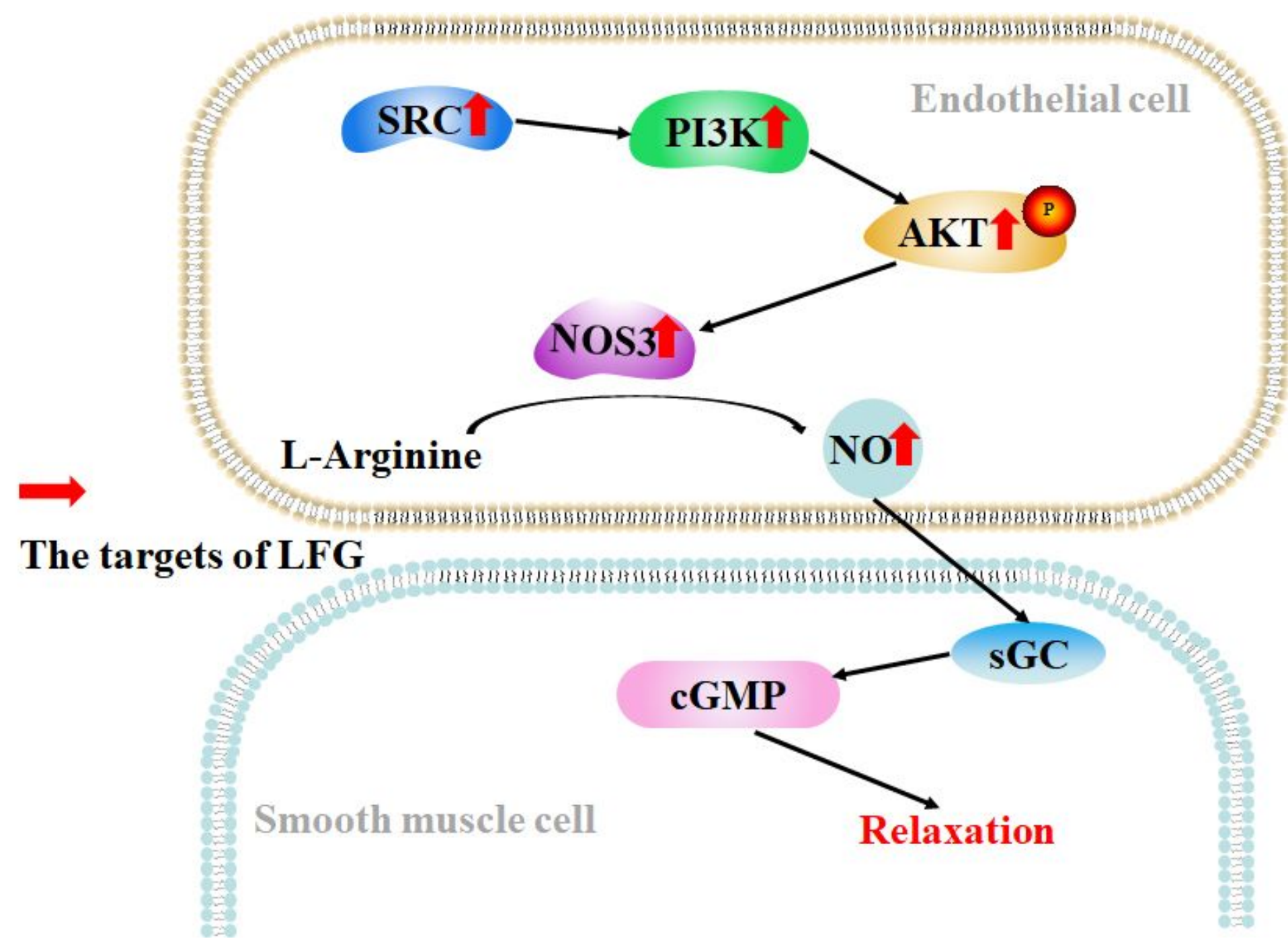

Figure 11

LFG antihypertensive mechanism.

\section{Supplementary Files}

This is a list of supplementary files associated with this preprint. Click to download.

- Additionalfile1.docx

- Additionalfile2.docx 